\title{
The Scaling of Arnol'd Tongues for Differentiable Homeomorphisms of the Circle*
}

\author{
Leo B. Jonker
}

Department of Mathematics and Statistics, Queen's University, Kingston, Ontario, Canada K7L 3N6

\begin{abstract}
We show both for diffeomorphisms of the circle and for differentiable homeomorphisms that are not diffeomorphisms, that the widths of the Arnol'd tongues in a one parameter family scale as $q^{-3}$ when $q$ is the denominator of the rotation number.
\end{abstract}

\section{Introduction}

When $f$ is a homeomorphisms of the circle $S^{1}=\mathbb{R} / \mathbb{Z}$ to itself, the rotation number $\rho(f)$ of $f$ constitutes an invariant that measures the rate at which the orbit of a point wraps around the circle. The concept originated with Poincare [9] and is best defined in terms of a lift $F$ of $f$ to the real line as

$$
\rho(f)=\lim _{n \rightarrow \infty} \frac{1}{n}\left(F^{n}(x)-x\right),
$$

where $x$ is any initial point. Both $F$ and the rotation number are defined uniquely up to translation by an integer. It is easy to see that as a function $C^{0}\left(S^{1}\right) \rightarrow \mathbb{R}, \rho(f)$ is continuous on the set of homeomorphisms, and that $f_{1}<f_{2}$ implies $\rho\left(f_{1}\right) \leqq \rho\left(f_{2}\right)$. Arnol'd in [2] was interested in the level sets of the function $\rho$ in the $(\lambda, \kappa)$-parameter plane of the "standard" function family

$$
F_{\lambda, \kappa}(x)=x+\lambda+(\kappa / 2 \pi) \sin (2 \pi x),
$$

giving rise to the so-called Arnol'd tongues. Since that time the universal attributes of such Arnol'd tongue pictures have been of considerable interest. The following is a consequence of the work of Herman [6]: Let $\sigma$ be the golden mean $(\sqrt{5}-1) / 2$ and let $p_{n} / q_{n}$ be the $n^{\text {th }}$ rational approximant of $\sigma$. For some fixed initial point $x_{0}$ and fixed $\kappa$ let $\lambda=\lambda_{n}$ represent the parameter value at which $x_{0}$ is periodic under $F_{\lambda, \kappa}$ with rotation number $p_{n} / q_{n}$, and let $\lambda_{0}$ be the parameter value at which $F_{\lambda, \kappa}$

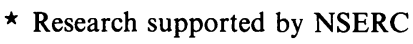


has rotation number $\sigma$. Then

$$
\lim _{n \rightarrow \infty}\left(\lambda_{n}-\lambda_{0}\right)^{n}=-\sigma^{-2}
$$

For circle maps which are homeomorphisms but not diffeomorphisms (e.g. $F_{\lambda, \kappa}$ with $\kappa=1$ ), work by Rand, Ostlund, Sethna and Siggia [10] gives numerical evidence for an analogous scaling at the golden mean. See also [8] in this connection.

Other results concern the "thickness" of the set of Arnol'd tongues at a fixed value of $k$. Herman in [6] proved the following result: If $f_{\lambda}$ is a $C^{1}$ family of $C^{r}$ orientation preserving diffeomorphisms, $r \geqq 3$, with $\rho\left(f_{0}\right) \neq \rho\left(f_{1}\right)$, then the set of parameter values for which the rotation number is irrational has positive Lebesgue measure. More recently, in [11] Świątek proved that the analogous result is false for homeomorphisms with critical points: Under rather general assumptions, which certainly admit the family $F_{\lambda, \kappa}$ with $\kappa=1$, he shows that the parameter values corresponding to rational rotation numbers constitute a set of full measure. This was previously conjectured and supported by numerical evidence in a paper by Jensen, Bak and Bohr [7]. In [1] Alstr $\phi \mathrm{m}$ reports on a numerical study of the dimension of the set of parameter values giving irrational rotation numbers for a family of one-to-one differentiable circle maps with critical points. Alstr $\phi \mathrm{m}$ conjectures that for a family of the form $F_{\lambda}(x)=F(x)+\lambda$, where $F$ is assumed to have just one critical point of order $r$, the limit capacity of the set of parameter values giving rise to irrational rotation numbers is equal to $r^{-1 / 8}$.

In [3] Cvitanović, Shraiman and Söderberg examine numerically the scaling of the widths of Arnol'd tongues for a family of critical maps $(\kappa=1)$. They find that if $p$ is a fixed integer and if $q \rightarrow \infty$, then the width of the Arnol'd tongue for the rotation number $p / q$ is asymptotic to $C q^{-3}$ for some constant C. A recent preprint by Ecke, Farmer and Umberger [4] examines numerically this scaling at a fixed value of the parameter $\kappa<1$. They find that the Arnol'd tongues in this case also satisfy the same scaling law.

The purpose of this paper is to prove that these conjectures are correct for generic families of circle maps, and for more general sequence of rational rotation numbers, obtained as successive mediants between nodes in the Farey tree. Our proofs do not yield the conclusion that the asymptotic estimate is uniform with respect to the natural rescaling associated with the Farey tree. For diffeomorphisms a uniform scaling estimate is obtained (by different methods) in a preprint of Graczyk [5]. We indicate as well that for non-generic families of circle maps other scalings can occur. Although experiments suggest it, we do not know whether the standard family satisfies the generic conditions required for our main scaling results.

\section{Statement of Results}

We consider families of homeomorphisms of the circle satisfying the following assumptions:

$F_{\lambda}$ belongs to the class $\mathscr{F}$ consisting of one-parameter function families satisfying the following hypotheses: 
(i) As a function of $x$ and $\lambda$ the function $F_{\lambda}(x)$ has continuous derivatives up to first order in $\lambda$ and $x$.

(ii) For each $\lambda$ in the parameter interval $T$ the function $F_{\lambda}$ is a homeomorphism of the circle.

(iii) If $\lambda$ is on the boundary of the set of parameter values for which $\rho\left(F_{\lambda}\right)=p / q$ for some rational $p / q$, and if $F_{\lambda}^{q}(x)=x+p$, then the $F_{\lambda}$-orbit of $x$ is the only periodic orbit, $\left(F_{\lambda}^{q}\right)^{\prime \prime}(x),(\partial / \partial \lambda)\left(\left(F_{\lambda}^{q}\right)^{\prime}(x)\right)$ and $(\partial / \partial \lambda)\left(\left(F_{\lambda}^{q}\right)^{\prime \prime}(x)\right)$ exist and are continuous on a neighbourhood of $(\lambda, x)$, and $\left(F_{\lambda}^{q}\right)^{\prime \prime}(x)$ is different from zero.

(iv) $(\partial / \partial \lambda) F_{\lambda}(x)>0$ for all $x \in \mathbb{R}$.

Condition (iii) is a condition that holds for a generic differentiable family of $C^{2}$ circle maps. It is easy to see that condition (iii) holds for the standard map with $q=1$. Whether it holds true in general for the standard map $I$ do not know. We also have the following more general condition.

(iii') If $\lambda$ is on the boundary of the set of parameter values for which $\rho\left(F_{\lambda}\right)=p / q$ for some rational number $p / q$, and if $F_{\lambda}^{q}(x)=x+p$, then the $F_{\lambda}$-orbit of $x$ is the only periodic orbit. Furthermore, if $t$ is the smallest integer greater than 1 for which $\left(F_{\lambda}^{q}\right)^{(t)}(x) \neq 0$, then $\left(F_{\lambda}^{q}\right)^{(t)}(x)$, and $(\partial / \partial \lambda)\left(\left(F_{\lambda}^{q}\right)^{(t)}(x)\right)$ exist and are continuous on a neighbourhood of $(\lambda, x)$.

We shall use $\mathscr{F}$, to denote the class of function families satisfying conditions (i), (ii), (iii') and (iv). Note that the value of $t$ in (iii') is necessarily even. We shall see below that the asymptotic behaviour for members of $\mathscr{F}^{\prime}$ is in general different from the behaviour observed in the computer studies cited above. Our main results are the following:

Theorem 1.1. Suppose $F_{\lambda}$ is a function family belonging to the class $\mathscr{F}$ and suppose $p / q<r / s$ [respectively $p / q>r / s]$ is a pair of Farey neighbours. For a fixed point $a \in \mathbb{R}$ let $\lambda_{0}$ be the largest [respectively smallest] parameter at which $\rho\left(F_{\lambda}\right)=p / q$ and let $\lambda_{n}$ be the unique parameter at which $F_{\lambda}^{n q+s}(a)=a+n p+r$. Then there are numbers $C_{1}$ and $C_{2}$ such that $C_{1}(n q+s)^{-2}<\left|\lambda_{n}-\lambda_{0}\right|<C_{2}(n q+s)^{-2}$ for all $n \geqq 1$.

Two rational numbers $p / q$ and $r / s$ are called Farey neighbours if $r q-p s= \pm 1$. This is equivalent to saying that $r / s$ and $p / q$ are adjacent in the set of rational numbers with denominators bounded above by the maximum of $q$ and $s$.

Theorem 1.2. Under the assumptions of Theorem 1.1, let $\theta_{n}=(n p+r) /(n q+s)$, and let $\Delta_{n}$ be the width of the parameter interval on which $\rho\left(F_{\lambda}\right)=\theta_{n}$. Then there are positive constants $C_{3}$ and $C_{4}$ such that $C_{3}(n q+s)^{-3}<\Delta_{n}<C_{4}(n q+s)^{-3}$ for all $n$.

In fact, it suffices to prove these theorems for the case where $p / q<r / s$ for this result can then be applied to the function family $-F_{-\lambda}(-x)$. Also, the conclusions of Theorems 1.1 and 1.2 for a specific rational number $p / q$ require assumption (iii) only for that specific $p / q$.

The theorems are both consequence of a study of the restriction of the iterates of the map $G_{\lambda}=F_{\lambda}^{q}-p$ to an interval $\left[a, G_{\lambda_{0}}(a)\right]$. For each iterate $G_{\lambda}^{n}$ we consider the values of $\lambda$ for which that iterate followed by $\left\{F_{\lambda}^{s}-r\right\}$ maps a to a point $w$ in the interval $\left[G_{\lambda_{0}}^{-1}(a), G_{\lambda_{0}}(a)\right]$. We refer to these functions as the $n^{\text {th }}$ return maps associated with the pair $\{p / q, r / s\}$. We have the following propositions regarding these return maps: 
Proposition 1.3. Let $I=\left[a, G_{\lambda_{0}}(a)\right]$, and let $w$ be a point in the interval $\left[G_{\lambda_{0}}^{-1}(a), G_{\lambda_{0}}(a)\right]$. Let $\lambda_{n}(w)$ be the unique parameter value for which the $n^{\text {th }}$ return map $k_{n, \lambda}=\left\{F_{\lambda}^{s}-r\right\} \circ$ $\left\{F_{\lambda}^{q}-p\right\}^{n}$ sends a to $w$. Then the sequence of functions $k_{n, \lambda_{n}}(w)$ is uniformly convergent to a function $k_{w}$ of I into $J=\left[G_{\lambda_{0}}^{-1}(a), G_{\lambda_{0}}^{2}(a)\right]$.

It will be noted in the proof of this theorem that the result is valid on the larger class $\mathscr{F}^{\prime}$. The next estimate is specific to $\mathscr{F}$. For a corresponding estimate on $\mathscr{F}^{\prime}$ the expression $\lambda^{-3 / 2}$ should be replaced by $\lambda^{(1 / t)-2}$, where $t-1$ is the order of contact between the graph of $F_{\lambda_{0}}^{q}$ and the line $y=x+p$ :

Proposition 1.4. Let $k_{n, \lambda}$ be as defined in the statement of Proposition 1.3, with $k_{n, \lambda}(I) \subset J$. Then there exist numbers $c_{1}$ and $c_{2}$ independent of $n$ such that

$$
c_{1} \lambda^{-3 / 2}<\frac{d}{d \lambda} k_{n, \lambda}(x)<c_{2} \lambda^{-3 / 2}
$$

for all $x \in I$.

To prove Theorems 1.1 and 1.2 we use the uniform approximability of $k_{n, \lambda_{n}(w)}$ by $k_{w}$ and the estimate of the derivative of $k_{n, \lambda}(a)$ given in Proposition 1.4 to estimate the size of the parameter interval required for the graph of $k_{n, \lambda}$ to cross the diagonal line $y=x$.

\section{Intermittency}

When $p / q$ and $r / s$ are a pair of Farey neighbours with $p / q<r / s$, then the sequence of numbers $\theta_{n}=(n p+r) /(n q+s)$ constitutes a sequence that converges to $p / q$. Thus the behaviour we are studying is in the parameter range for which the graph of the function $F_{\lambda}^{q}-p$ lies just above the diagonal line $y=x$. The behaviour of the return map $k_{n, \lambda}$ is therefore dominated by the large number of iterates $\left(F_{\lambda}^{q}-p\right)^{n}(I)$ located near the location where $F_{\lambda_{0}}^{q}-p$ had a fixed point. This phenomenon is known as intermittency. The two lemmas in this section serve to show that the effect of this intermittency on the return map is uniform if interpreted in the right way.

Lemma 2.1. Suppose $F_{\lambda}$ is a family of functions belonging to the class $\mathscr{F}^{\prime}$ or to the class $\mathscr{F}$, and suppose that 0 is the largest parameter value such that $\rho\left(F_{\lambda}\right)=0$, and that $F_{0}(0)=0$. Then for any $\delta>1$ and $m \in \mathbb{Z}_{+}$there exist numbers $\tau>0$ as small as desired, and $\varepsilon>0$ such that for $0<\lambda<\varepsilon$ we have $F_{\lambda}^{m}(-\tau)<0$ and $F_{\lambda}^{-m}(\tau)>0$, and for any $x \in \mathbb{R}$ and $q \in \mathbb{Z}_{+}$with

$$
x \in\left[-\tau, F_{\lambda}^{m}(-\tau)\right)
$$

and

$$
F_{\lambda}^{q}(x) \in\left[F_{\lambda}^{-m}(\tau), \tau\right)
$$

we have

$$
\frac{1}{\delta}<\left(F_{\lambda}^{q}\right)^{\prime}(x)<\delta
$$

Proof. Let $\phi_{\lambda}(x)=F_{\lambda}(x)-x$ and suppose the $t^{\text {th }}$ is the first non-vanishing derivative of $\phi_{0}$ at $x=0$. Let $\phi_{0}^{(t)}(0)=(t !) \alpha$. It is easy to see that $t$ must be even. Choose $\delta_{1}>1$ 
so that $\delta_{1}^{7}<\delta$. Also choose $\alpha_{1}$ and $\alpha_{2}$ so that $0<\alpha_{1}<\alpha<\alpha_{2}$, and $1 / \delta_{1}<\alpha_{1} / \alpha_{2}<$ $\alpha_{2} / \alpha_{1}<\delta_{1}$. Choose $\sigma>0$ so that for $x \in[-\sigma, \sigma]$ we have

$$
t ! \alpha_{1}<\phi_{0}^{(t)}(x)<t ! \alpha_{2} \text {. }
$$

Integrating this $t$ times gives

$$
\alpha_{1} x^{t} \leqq \phi_{0}(x) \leqq \alpha_{2} x^{t}
$$

Now choose $\tau>0$ so that $F_{0}^{2}(-\sigma)<-\tau$ and $F_{0}^{-1}(\sigma)>\tau$. Furthermore, by the Mean Value Theorem, $\tau / F_{0}^{-m-2}(\tau)$ is the product of the derivatives of $F_{0}$ at $m-2$ points in the interval $(0, \tau)$. Therefore, by picking $\tau>0$ small enough we may assume that

$$
\left(\frac{\tau}{F_{0}^{-m-2}(\tau)}\right)^{t}<\delta_{1}
$$

Using (2.1.1) we conclude that if $u, v \in\left[F_{0}^{-m-2}(\tau), \tau\right]$ then

$$
\frac{\alpha_{1}}{\alpha_{2}} \cdot \frac{1}{\delta_{1}}<\frac{\phi_{0}\left(F_{0}^{-m-2}(\tau)\right)}{\phi_{0}(\tau)}<\frac{\phi_{0}(u)}{\phi_{0}(v)}<\frac{\phi_{0}(\tau)}{\phi_{0}\left(F_{0}^{-m-2}(\tau)\right)}<\frac{\alpha_{2}}{\alpha_{1}} \delta_{1} .
$$

Thus

$$
\frac{1}{\delta_{1}^{2}}<\frac{\phi_{0}(u)}{\phi_{0}(v)}<\delta_{1}^{2} .
$$

Similarly, by choosing $\tau>0$ small enough, we may assume that if $u, v \in$ $\left[F_{0}^{-2}(-\tau), F_{0}^{m+1}(-\tau)\right]$ then $(2.1 .2)$ holds. Note as well that from (2.1.1) we have

$$
\frac{1}{\delta_{1}}<\frac{\phi_{0}(\tau)}{\phi_{0}(-\tau)}<\delta_{1}
$$

By continuity we may now pick $\varepsilon>0$ so small that for all $\lambda \in(0, \varepsilon)$,

$$
\left[F_{\lambda}^{-1}(-\tau), F_{\lambda}^{m}(-\tau)\right] \subseteq\left[F_{0}^{-2}(-\tau), F_{0}^{m+1}(-\tau)\right]
$$

and

$$
\left[F_{\lambda}^{-m-1}(\tau), \tau\right] \subseteq\left[F_{0}^{-m-2}(\tau), \tau\right] .
$$

Since $\phi_{\lambda}$ converges uniformly to $\phi_{0}$ it follows from (2.1.2) and (2.1.3) that we may now choose $\varepsilon>0$ even smaller, if necessary, to ensure that for $u, v \in\left[F_{\lambda}^{-m-1}(\tau), \tau\right]$ as well as for $u, v \in\left[F_{\lambda}^{-1}(-\tau), F_{\lambda}^{m}(-\tau)\right]$ we have

$$
\frac{1}{\delta_{1}^{3}}<\frac{\phi_{\lambda}(u)}{\phi_{\lambda}(v)}<\delta_{1}^{3}
$$

and

$$
\frac{1}{\delta_{1}}<\frac{\phi_{\lambda}(\tau)}{\phi_{\lambda}(-\tau)}<\delta_{1}
$$

Now suppose $x \in\left[-\tau, F_{\lambda}^{m}(-\tau)\right)$ and $F_{\lambda}^{q}(x) \in\left[F_{\lambda}^{-m}(\tau), \tau\right)$. Let $x_{i}=F_{\lambda}^{i}(x)$ and $a_{i}=\phi_{\lambda}\left(x_{i}\right)$ for $i=-1,0,1, \ldots, q$. Using the Mean Value Theorem and the fact that $F_{\lambda}^{\prime \prime}(u) \geqq 0$ 
on $[-\sigma, \sigma]($ see $(2.1 .1))$ we have for $i=-1,0,1, \ldots, q$,

$$
\frac{a_{i}}{a_{i-1}}<F_{\lambda}^{\prime}\left(x_{i}\right)<\frac{a_{i+1}}{a_{i}} \text {. }
$$

Therefore

$$
\frac{a_{q-1}}{a_{-1}}<\left(F_{\lambda}^{q}\right)^{\prime}(x)<\frac{a_{q}}{a_{0}}
$$

But by (2.1.4),

$$
\frac{1}{\delta_{1}^{3}}<\frac{a_{q}}{\phi_{\lambda}(\tau)}, \quad \frac{a_{q-1}}{\phi_{\lambda}(\tau)}<\delta_{1}^{3}
$$

and

$$
\frac{1}{\delta_{1}^{3}}<\frac{\phi_{\lambda}(-\tau)}{a_{-1}}, \quad \frac{\phi_{\lambda}(-\tau)}{a_{0}}<\delta_{1}^{3} .
$$

Combining (2.1.5), (2.1.6), (2.1.7), and (2.1.8) we get

$$
\frac{1}{\delta}<\frac{1}{\delta_{1}^{7}}<\left(F_{\lambda}^{q}\right)^{\prime}(x)<\delta_{1}^{7}<\delta .
$$

If the function family is of the form $F_{\lambda}(x)=F(x)+\lambda$, then it is easy to see that if $x_{i}=F_{\lambda}^{i}(x)$, then $(d / d \lambda) F_{\lambda}^{q}(x)=1+F_{\lambda}^{\prime}\left(x_{q-1}\right)+F_{\lambda}^{\prime}\left(x_{q-1}\right) F_{\lambda}^{\prime}\left(x_{q-2}\right)+\cdots+F_{\lambda}^{\prime}\left(x_{q-1}\right) \cdots \cdots$ $F_{\lambda}^{\prime}\left(x_{1}\right)$. While this formula is not correct for a general family, we shall need to estimate it in order to obtain an estimate for $(d / d x) F_{\lambda}^{q}(x)$ in the general case. The next two lemmas serve to define a constant $L_{t}$ needed in this estimate.

Lemma 2.2. For any $\tau>0$ and $k \in \mathbf{Z}_{+}$,

$$
0<\lim _{a \rightarrow 0^{+}} a^{1-(1 / 2 k)} \int_{-\tau}^{\tau} \frac{d x}{a+x^{2 k}}<\infty .
$$

Proof. Factoring the denominator $a+x^{2 k}$ (over $\mathbb{C}$ ) and using partial fractions and the principal branch of $\ln z$, we get

$$
\int_{0}^{\tau} \frac{d x}{a+x^{2 k}}=\sum_{m=1}^{2 k} c_{m} \ln \left(1-\frac{\tau}{\gamma \omega^{m}}\right)
$$

where $c_{m}=-\left(a^{(1 / 2 k)-1}\right) \cdot \omega^{m} \alpha /(2 k-1)$, and where $\alpha=e^{i \pi / 2 k}, \gamma=\alpha \cdot a^{1 / 2 k}$, and $\omega=e^{i \pi / k}$. Therefore,

$$
\begin{aligned}
& \lim _{a \rightarrow 0^{+}} a^{1-(1 / 2 k)} \int_{0}^{\tau} \frac{d x}{a+x^{2 k}} \\
& \quad=\lim _{a \rightarrow 0^{+}} \frac{-\alpha}{2 k-1} \sum_{m=1}^{2 k} \omega^{m} \ln \left(1-\frac{\tau}{\gamma \tau^{m}}\right) \\
& \quad=\lim _{a \rightarrow 0^{+}} \frac{-\alpha}{2 k-1} \sum_{m=1}^{2 k} \omega^{m} \ln \left(\frac{\gamma}{\tau}-\frac{1}{\omega^{m}}\right)=-\frac{-\alpha}{2 k-1} \sum_{m=1}^{2 k} \omega^{m} \ln \left(-1 / \omega^{m}\right) .
\end{aligned}
$$


Corollary 2.3. $\int_{-\tau}^{\tau} \frac{d x}{\left(a+x^{2 k}\right)^{2}}$ is of order $a^{(1 / 2 k)-2}$ as a goes to zero.

Proof. Let $L$ be the limit obtained in the preceding lemma. Then

by l'Hôpital's Rule.

$$
2 L=\lim _{a \rightarrow 0^{+}} a^{1-(1 / 2 k)} \int_{-\tau}^{\tau} \frac{d x}{a+x^{2 k}}=\lim _{a \rightarrow 0^{+}} \frac{a^{2-(1 / 2 k)}}{\left(1-\frac{1}{2 k}\right)^{\tau}} \int_{-\tau}^{\tau} \frac{d x}{\left(a+x^{2 k}\right)^{2}},
$$

For an even positive integer we define the constant $L_{t}$ by

$$
L_{t}=\lim _{a \rightarrow 0^{+}} a^{2-(1 / t)} \int_{-\tau}^{\tau} \frac{d x}{\left(a+x^{t}\right)^{2}} .
$$

Lemma 2.4. Suppose $F_{\lambda}$ is a family of functions belonging to the class $\mathscr{F}^{\prime}$ such that 0 is the largest parameter value at which $\rho(\lambda)=0$, and such that $F_{0}(0)=0$. Suppose that $t$ is the smallest integer greater than 1 for which $F_{0}^{(t)}(0) \neq 0$. Let $(t !) \alpha=F_{0}^{(t)}(0)$, let $\beta=\left.(d / d \lambda) F_{\lambda}(0)\right|_{\lambda=0}$ and let $K_{0}=\alpha^{1-(1 / t)} \beta^{(1 / t)-2} \cdot L_{t}$. Then for any $\delta>1$ and $m \in \mathbb{Z}_{+}$ there exist numbers $\tau>0$, as small as desired, and $\varepsilon>0$ such that for $0<\lambda<\varepsilon$ we have $F_{\lambda}^{m}(-\tau)<0$ and $F_{\lambda}^{-m}(\tau)>0$, and for any $x \in \mathbb{R}$ and $q \in \mathbb{Z}_{+}$such that

$$
x \in\left[-\tau, F_{\lambda}^{m}(-\tau)\right) \text { and } F_{\lambda}^{q}(x) \in\left[F_{\lambda}^{-m}(\tau), \tau\right),
$$

we have

$$
\begin{aligned}
\frac{1}{\delta} K_{0} \tau^{t} \lambda^{(1 / t)-2} & <1+F_{\lambda}^{\prime}\left(x_{q-1}\right)+F_{\lambda}^{\prime}\left(x_{q-1}\right) F_{\lambda}^{\prime}\left(x_{q-2}\right)+\cdots+F_{\lambda}^{\prime}\left(x_{q-1}\right) \cdots \cdot F_{\lambda}^{\prime}\left(x_{1}\right) \\
& <\delta K_{0} \tau^{t} \lambda^{(1 / t)-2} .
\end{aligned}
$$

Proof. Choose numbers $\delta_{1}, \delta_{2}, \delta_{3}, \delta_{4}$ so that $1<\delta_{1}<\delta_{2}<\delta_{3}<\delta_{4}<\delta$. Then choose $\varepsilon_{1}>0$ such that

$$
\begin{gathered}
\delta_{3} \frac{1}{1-2 \varepsilon_{1}\left(\frac{1}{1-\varepsilon_{1}}\right)^{3}}<\delta_{4}, \\
\frac{1}{\delta_{3}} \frac{1}{1+2 \varepsilon_{1}\left(\frac{1}{1-\varepsilon_{1}}\right)^{3}}>\frac{1}{\delta_{4}}, \\
\delta_{4}\left(1+\varepsilon_{1}\right)<\delta,
\end{gathered}
$$

and

$$
\frac{1}{\delta_{4}} \cdot \frac{1}{1+\varepsilon_{1}}>\frac{1}{\delta}
$$

By Corollary 2.3, with $k=1$, we have that if $\alpha_{i}$ and $\beta_{i}$ are positive reals, then

$$
\lim _{\lambda \rightarrow 0^{+}} \lambda^{2-(1 / t)} \int_{-\tau}^{\tau} \frac{d x}{\left(\beta_{i} \lambda+\alpha_{i} x^{t}\right)^{2}}=\alpha_{i}^{-1 / t} \cdot \beta_{i}^{(1 / t)-2} \cdot L_{t},
$$

where $L_{t}$ is independent of $\alpha_{i}$ and $\beta_{i}$. 
Now let $K_{1}=\alpha^{-1 / t} \cdot \beta^{(1 / t)-2} \cdot L_{t}$, and choose $\alpha_{1}, \alpha_{2}, \beta_{1}, \beta_{2}$ so that $0<\alpha_{1}<\alpha<\alpha_{2}$ and $0<\beta_{1}<\beta<\beta_{2}$, and so that

$$
\begin{gathered}
\frac{1}{\delta_{1}} K_{1}<\alpha_{2}^{-1 / t} \cdot \beta_{2}^{(1 / t)-2} \cdot L_{t}, \\
\alpha_{1}^{-1 / t} \cdot \beta_{1}^{(1 / t)-2} \cdot L_{t}<\delta_{1} K_{1}, \\
\delta_{4} \alpha_{2}<\delta \alpha
\end{gathered}
$$

and

$$
\frac{1}{\delta_{4}} \alpha_{1}>\frac{1}{\delta} \alpha
$$

Let $\phi_{\lambda}(x)=F_{\lambda}(x)-x$, and choose $\sigma>0$ so that $F_{0}^{(t)}(x)>0$,

$$
\alpha_{1} x^{t}<\phi_{0}(x)<\alpha_{2} x^{t}
$$

and

$$
\left|\phi_{0}^{\prime}(x)\right|<\varepsilon_{1}
$$

for all $x \in[-\sigma, \sigma]$, and choose $\tau>0$ such that

$$
F_{0}^{-1}(-\tau)>-\sigma \text { and } F_{0}(\tau)<\sigma .
$$

We now choose $\varepsilon>0$ so that when $\lambda \in(0, \varepsilon)$, then $F_{\lambda}^{m}(-\tau)<0$, and $F_{\lambda}^{-m}(\tau)>0$, and for all $x \in[-\sigma, \sigma]$, we have

$$
\begin{gathered}
F_{\lambda}^{(t)}(x)>0, \quad \beta_{1} \lambda+\alpha_{1} x^{t}<\phi_{\lambda}(x)<\beta_{2} \lambda+\alpha_{2} x^{t}, \\
\frac{\delta_{1}}{\delta_{2}}<A / B<\frac{\delta_{2}}{\delta_{1}}
\end{gathered}
$$

where for $i=1,2$,

$$
A=\lambda^{2-(1 / t)} \int_{-\tau}^{\tau} \frac{d x}{\left(\beta_{i} \lambda+\alpha_{i} x^{t}\right)^{2}}
$$

and

$$
B=\alpha_{i}^{-1 / t} \cdot \beta_{i}^{(1 / t)-2} \cdot L_{t}, \quad\left|\phi_{\lambda}^{\prime}(x)\right|<\varepsilon_{1},
$$

and so that

$$
\frac{1}{\delta_{3}} K_{1}<\frac{1}{\delta_{2}} K_{1}-\lambda^{2-(1 / t)} \sigma\left\{\frac{1}{\left(1-\varepsilon_{1}\right)^{m+1}}+1\right\} \frac{1}{\left(\beta_{1} \lambda+\alpha_{1} \tau^{t}\right)^{2}},
$$

and

$$
\delta_{3} K_{1}>\delta_{2} K_{1}+\lambda^{2-(1 / t)} \sigma\left\{\frac{1}{\left(1-\varepsilon_{1}\right)^{m+1}}+1\right\} \frac{1}{\left(\beta_{1} \lambda+\alpha_{1} \tau^{t}\right)^{2}} .
$$

Furthermore, using (2.4.7) and (2.4.8) we may assume that

$$
\delta_{4}\left(\beta_{2} \lambda+\alpha_{2} \tau^{t}\right)<\delta \alpha \tau^{t}
$$




$$
\frac{1}{\delta_{4}}\left(\beta_{1} \lambda+\alpha_{1} \tau^{t}\right)>\frac{1}{\delta} \alpha \tau^{t}
$$

when $\lambda \in(0, \varepsilon)$.

We will later need an estimate for $\int_{-\tau}^{\tau} \frac{d x}{\left(\phi_{\lambda}(x)\right)^{2}}$ : Combining (2.4.12), (2.4.13), (2.4.5) and (2.4.6), we have

$$
\lambda^{(1 / t)-2} \cdot \frac{1}{\delta_{2}} \cdot K_{1}<\int_{-\tau}^{\tau} \frac{d x}{\left(\phi_{\lambda}(x)\right)^{2}}<\lambda^{(1 / t)-2} \cdot \delta_{2} \cdot K_{1} .
$$

For the remainder of the proof assume that $x \in\left[-\tau, F_{\lambda}^{m}(-\tau)\right)$ and $F_{\lambda}^{q}(x) \in\left[F_{\lambda}^{-m}(\tau), \tau\right)$. Let $x_{i}=F_{\lambda}^{i}(x)$ and $a_{i}=\phi_{\lambda}\left(x_{i}\right)$ for $i=-1,0,1,2, \ldots, q+1$. Also let $X$ denote the sum $1+F_{\lambda}^{\prime}\left(x_{q-1}\right)+F_{\lambda}^{\prime}\left(x_{q-1}\right) F_{\lambda}^{\prime}\left(x_{q-2}\right)+\cdots+F_{\lambda}^{\prime}\left(x_{q-1}\right) \cdots \cdot F_{\lambda}^{\prime}\left(x_{1}\right)$. Then by the mean value theorem, for $i=0,1,2, \ldots, q-1$, we have

$$
\frac{a_{i}}{a_{i-1}}<F_{\lambda}^{\prime}\left(x_{i}\right)<\frac{a_{i+1}}{a_{i}}
$$

Therefore,

$$
a_{q-1}\left\{\frac{1}{a_{q-1}}+\frac{1}{a_{q-2}}+\cdots+\frac{1}{a_{0}}\right\}<X<a_{q}\left\{\frac{1}{a_{q}}+\frac{1}{a_{q-1}}+\cdots+\frac{1}{a_{1}}\right\} .
$$

We now proceed to estimate $a_{q}\left\{\left(1 / a_{q}\right)+\left(1 / a_{q-1}\right)+\cdots+\left(1 / a_{1}\right)\right\}$, by writing it as

$$
\phi_{\lambda}\left(x_{q}\right) \cdot \sum_{i=1}^{q}\left(\frac{1}{\phi_{\lambda}\left(x_{i}\right)}\right)^{2} \phi_{\lambda}\left(x_{i}\right) \text {. }
$$

Since $\phi_{\lambda}\left(x_{i}\right)=x_{i+1}-x_{i}=\Delta x_{i}$, this is a Riemann sum for the function $\left(1 /\left(\phi_{\lambda}\left(x_{i}\right)\right)\right)^{2}$ on the interval $\left[x_{1}, x_{q+1}\right]$. Note that when $v \in\left(u, F_{\lambda}(u)\right] \subset[-\sigma, \sigma]$, then

$$
\phi_{\lambda}(v)=\phi_{\lambda}^{\prime}(w)(v-u)+\phi_{\lambda}(u)
$$

for some $w \in(u, v)$. Thus

$$
\frac{\phi_{\lambda}(v)}{\phi_{\lambda}(u)}=\phi_{\lambda}^{\prime}(w) \frac{v-u}{\phi_{\lambda}(u)}+1
$$

Here

$$
\left|\phi_{\lambda}^{\prime}(w) \frac{v-u}{\phi_{\lambda}(u)}\right|<\varepsilon_{1}
$$

by (2.4.14), and so, whenever $v \in\left[u, F_{\lambda}(u)\right] \subset[-\sigma, \sigma]$, we have

$$
1-\varepsilon_{1}<\left|\frac{\phi_{\lambda}(v)}{\phi_{\lambda}(u)}\right|<1+\varepsilon_{1} .
$$

Now let $u_{i}$ and $v_{i}$ be points in $\left[x_{i}, x_{i+1}\right]$, where $\phi_{\lambda}(u)$ reaches its maximum and 
minimum values respectively. Then

$$
\begin{array}{rlrl}
\left|\int_{x_{i}}^{x_{i+1}} \frac{1}{\left(\phi_{\lambda}(u)\right)^{2}} d u-\left(\frac{1}{\phi_{\lambda}\left(x_{i}\right)}\right)^{2} \Delta x_{i}\right| & <\phi_{\lambda}\left(x_{i}\right)\left|\frac{1}{\left(\phi_{\lambda}\left(v_{i}\right)\right)^{2}}-\frac{1}{\left(\phi_{\lambda}\left(u_{i}\right)\right)^{2}}\right| \\
& =\phi_{\lambda}\left(x_{i}\right)\left|\frac{2 \phi_{\lambda}^{\prime}\left(z_{i}\right)}{\left(\phi_{\lambda}\left(z_{i}\right)\right)^{3}}\right| \cdot\left|u_{i}-v_{i}\right| \quad \text { for some } \quad z_{i} \in\left[x_{i}, x_{i+1}\right] \\
& <\phi_{\lambda}\left(x_{i}\right)^{2}\left|\frac{2 \phi_{\lambda}^{\prime}\left(z_{i}\right)}{\left(\phi_{\lambda}\left(z_{i}\right)\right)^{3}}\right|<\left(\frac{1}{1-\varepsilon_{1}}\right)^{3} \frac{\left|2 \phi_{\lambda}^{\prime}\left(z_{i}\right)\right|}{\phi_{\lambda}\left(x_{i}\right)} & \text { by (2.4.22) } \\
& <2 \varepsilon_{1}\left(\frac{1}{1-\varepsilon_{1}}\right)^{3} \frac{1}{\phi_{\lambda}\left(x_{i}\right)}, & \text { by (2.4.14). }
\end{array}
$$

Thus,

$$
\left|\int_{x_{1}}^{x_{q}+1} \frac{1}{\left(\phi_{\lambda}(u)\right)^{2}} d u-\sum_{i=1}^{q} \frac{1}{\phi_{\lambda}\left(x_{i}\right)}\right|<2 \varepsilon_{1}\left(\frac{1}{1-\varepsilon_{1}}\right)^{3} \sum_{i=1}^{q} \frac{1}{\phi_{\lambda}\left(x_{i}\right)} .
$$

Similarly, we get

$$
\left|\int_{x_{0}}^{x_{g}} \frac{1}{\left(\phi_{\lambda}(u)\right)^{2}} d u-\sum_{i=0}^{q-1} \frac{1}{\phi_{\lambda}\left(x_{i}\right)}\right|<2 \varepsilon_{1}\left(\frac{1}{1-\varepsilon_{1}}\right)^{3} \sum_{i=0}^{q-1} \frac{1}{\phi_{\lambda}\left(x_{i}\right)} .
$$

We now compare these integrals to the integral from $-\tau$ to $\tau$,

$$
\begin{aligned}
& \left|\int_{x_{1}}^{x_{q}+1}\left(\frac{1}{\phi_{\lambda}(u)}\right)^{2} d u-\int_{-\tau}^{\tau}\left(\frac{1}{\phi_{\lambda}(u)}\right)^{2} d u\right| \\
& \quad \leqq\left|\int_{-\tau}^{x_{1}}\left(\frac{1}{\phi_{\lambda}(u)}\right)^{2} d u\right|+\left|\int_{\tau}^{x_{q}+1}\left(\frac{1}{\phi_{\lambda}(u)}\right)^{2} d u\right| \\
& \quad \leqq\left|x_{1}+\tau\right|\left(\frac{1}{\phi_{\lambda}\left(x_{1}\right)}\right)^{2}+\left|x_{q+1}-\tau\right|\left(\frac{1}{\phi_{\lambda}(\tau)}\right)^{2} \\
& \quad \leqq\left|\sum_{i=0}^{m} \phi_{\lambda}\left(F_{\lambda}^{i}(-\tau)\right)\right|\left(\frac{1}{\phi_{\lambda}\left(x_{1}\right)}\right)^{2}+\left|\sum_{i=1}^{m+1} \phi_{\lambda}\left(F_{\lambda}^{-i}(\tau)\right)\right|\left(\frac{1}{\phi_{\lambda}(\tau)}\right)^{2} \\
& \quad<\frac{\sigma}{\left(1-\varepsilon_{1}\right)^{m+1}\left(\phi_{\lambda}(-\tau)\right)^{2}}+\frac{\sigma}{\left(\phi_{\lambda}(\tau)\right)^{2}} \text { by }(2.4 .22), \\
& \quad<\left\{\frac{\sigma}{\left.\left(1-\varepsilon_{1}\right)^{m+1}+\sigma\right\} \frac{1}{\left(\beta_{1} \lambda+\alpha_{1} \tau^{t}\right)^{2}},}\right.
\end{aligned}
$$

by (2.2.12). Combining (2.2.25) and (2.2.19), and using (2.4.15) and (2.2.16), we get

$$
\lambda^{(1 / t)-2} \cdot \frac{1}{\delta_{3}} \cdot K_{1}<\int_{x_{1}}^{x_{q+1}} \frac{d x}{\left(\phi_{\lambda}(x)\right)^{2}}<\lambda^{(1 / t)-2} \cdot \delta_{3} \cdot K_{1} .
$$

By a similar argument,

$$
\lambda^{(1 / t)-2} \cdot \frac{1}{\delta_{3}} \cdot K_{1}<\int_{x_{0}}^{x_{q}} \frac{d x}{\left(\phi_{\lambda}(x)\right)^{2}}<\lambda^{(1 / t)-2} \cdot \delta_{3} \cdot K_{1} .
$$


We are now ready to combine (2.4.26) and (2.4.23): Letting

$$
I=\int_{x_{1}}^{x_{q}+1} \frac{d x}{\left(\phi_{\lambda}(x)\right)^{2}}, \text { and } S=\sum_{i=1}^{q} \frac{1}{\phi_{\lambda}\left(x_{i}\right)},
$$

we have from (2.4.23)

$$
|I-S|<2 \varepsilon_{1}\left(\frac{1}{1-\varepsilon_{1}}\right)^{3} S
$$

whence

$$
\frac{I}{1+2 \varepsilon_{1}\left(1-\varepsilon_{3}\right)^{-3}}<S<\frac{I}{1-2 \varepsilon_{1}\left(1-\varepsilon_{3}\right)^{-3}} .
$$

Using (2.4.26) this gives

$$
\frac{1}{\delta_{3}} K_{1} \frac{\lambda^{(1 / t)-2}}{1+2 \varepsilon_{1}\left(1-\varepsilon_{3}\right)^{-3}}<S<\delta_{3} K_{1} \frac{\lambda^{(1 / t)-2}}{1-2 \varepsilon_{1}\left(1-\varepsilon_{3}\right)^{-3}} .
$$

Using (2.4.1) and (2.4.2) this leads to

$$
\lambda^{(1 / t)-2} \cdot \frac{1}{\delta_{4}} \cdot K_{1}<\sum_{i=1}^{q} \frac{1}{\phi_{\lambda}\left(x_{i}\right)}<\lambda^{(1 / t)-2} \cdot \delta_{4} \cdot K_{1} .
$$

Similarly,

$$
\lambda^{(1 / t)-2} \cdot \frac{1}{\delta_{4}} \cdot K_{1}<\sum_{i=0}^{q-1} \frac{1}{\phi_{\lambda}\left(x_{i}\right)}<\lambda^{(1 / t)-2} \cdot \delta_{4} \cdot K_{1} .
$$

Thus,

$$
a_{q}\left\{\frac{1}{a_{q}}+\frac{1}{a_{q-1}}+\cdots+\frac{1}{a_{1}}\right\}<\lambda^{(1 / t)-2} \cdot \delta_{4} \cdot K_{1} \cdot \phi_{\lambda}\left(x_{q}\right) .
$$

Now let $K=K_{1} \alpha$. Then, using (2.4.12), and (2.4.17), we obtain

$$
a_{q}\left\{\frac{1}{a_{q}}+\frac{1}{a_{q-1}}+\cdots \pm \frac{1}{a_{1}}\right\}<\lambda^{(1 / t)-2} \cdot \delta_{4} \cdot K_{1} \cdot\left(\beta_{2} \lambda+\alpha_{2} \tau^{t}\right)<\delta \cdot \lambda^{(1 / t)-2} \cdot K \cdot \tau^{t} .
$$

Similarly,

$$
\frac{1}{\delta} \cdot \lambda^{(1 / t)-2} \cdot K \cdot \tau^{t}<a_{q-1}\left\{\frac{1}{a_{q-1}}+\cdots+\frac{1}{a_{0}}\right\} .
$$

When we combine this with (2.4.21) we obtain the desired inequality.

We will now use Lemma 2.4 to obtain an estimate for $(d / d \lambda)\left(F_{\lambda}^{q}(x)\right)$ in a region of intermittency:

Lemma 2.5. Assume the hypotheses of Lemma 2.4, and let $\alpha=F_{0}^{(t)}(0), \beta=\left.(d / d \lambda) F_{\lambda}(0)\right|_{\lambda=0}$ and $K=L_{t} \cdot(\alpha / \beta)^{1-(1 / t)}$. Suppose $\delta>1$, and that $\tau>0$ and $\varepsilon>0$ are sufficiently small. Then for $0<\lambda<\varepsilon$ and for $x \in \mathbb{R}$ and $q \in \mathbb{Z}_{+}$such that

$$
x \in\left[-\tau, F_{\lambda}^{m}(-\tau)\right)
$$


and

$$
F_{\lambda}^{q}(x) \in\left[F_{\lambda}^{-m}(\tau), \tau\right)
$$

we have

$$
\frac{1}{\delta} \lambda^{(1 / t)-2} \cdot K \cdot \tau^{t}<\frac{d}{d \lambda}\left(F_{\lambda}^{q}(x)\right)<\delta \cdot \lambda^{(1 / t)-2} \cdot K \cdot \tau^{t}
$$

Proof. Choose $\delta_{0}$ so that $1<\delta_{0}<\delta$. Now choose $\beta_{1}$ and $\beta_{2}$ so that $0<\beta_{1}<\beta<\beta_{2}$ and so that

$$
\beta / \delta<\beta_{1} / \delta_{0}
$$

and

$$
\beta_{2} \delta_{0}<\beta \delta
$$

By Lemma 2.4 there exists numbers $\tau, \varepsilon$, and $K_{0}$ such that $\lambda \in(0, \varepsilon), x \in\left[-\tau, F_{\lambda}^{m}(-\tau)\right)$ and $F_{\lambda}^{q}(x) \in\left[F_{\lambda}^{-m}(\tau), \tau\right)$ imply, with $x_{i}=F_{\lambda}^{i}(x)$ as before,

$$
\begin{aligned}
\frac{1}{\delta_{0}} K_{0} \tau^{t} \lambda^{(1 / t)-2} & <1+F_{\lambda}^{\prime}\left(x_{q-1}\right)+\cdots+F_{\lambda}^{\prime}\left(x_{q-1}\right) \cdots \cdot F_{\lambda}^{\prime}\left(x_{1}\right) \\
& <\delta_{0} K_{0} \tau^{t} \lambda^{(1 / t)-2} .
\end{aligned}
$$

Since $(d / d \lambda) F_{\lambda}(u)$ is continuous in $(\lambda, u)$ jointly, we may assume that $\varepsilon$ and $\tau$ are so small that

$$
\beta_{1}<\frac{d}{d \lambda} F_{\lambda}(u)<\beta_{2}
$$

for all $u \in[-\tau, \tau)$ and all $\lambda \in(0, \varepsilon)$. Now

$$
\frac{d}{d \lambda} F_{\lambda}^{q}(x)=\left(\frac{d}{d \lambda} F_{\lambda}\right)\left(F_{\lambda}^{q-1}(x)\right)+F_{\lambda}^{\prime}\left(F_{\lambda}^{q-1}(x)\right) \frac{d}{d \lambda} F_{\lambda}^{q-1}(x),
$$

whence

$$
\beta_{1}+F_{\lambda}^{\prime}\left(x_{q-1}\right) \frac{d}{d \lambda} F_{\lambda}^{q-1}(x)<\frac{d}{d \lambda} F_{\lambda}^{q}(x)<\beta_{2}+F_{\lambda}^{\prime}\left(x_{-1}^{q}\right) \frac{d}{d \lambda} F_{\lambda}^{q-1}(x) .
$$

Repeating the same estimate for $(d / d \lambda) F_{\lambda}^{q-1}(x),(d / d \lambda) F_{\lambda}^{q-2}(x), \ldots$ we get

$$
\begin{gathered}
\beta_{1}\left\{1+F_{\lambda}^{\prime}\left(x_{q-1}\right)+\cdots+F_{\lambda}^{\prime}\left(x_{q-1}\right) \cdots \cdots F_{\lambda}^{\prime}\left(x_{1}\right)\right\}<\frac{d}{d \lambda} F_{\lambda}^{q}(x) \\
<\beta_{2}\left\{1+F_{\lambda}^{\prime}\left(x_{q-1}\right)+\cdots+F_{\lambda}^{\prime}\left(x_{q-1}\right) \cdot \cdots \cdot F_{\lambda}^{\prime}\left(x_{1}\right)\right\} .
\end{gathered}
$$

Using (2.5.3) this gives

$$
\beta_{1} \frac{1}{\delta_{0}} K_{0} \tau^{t} \lambda^{(1 / t)-2}<\frac{d}{d \lambda}\left(F_{\lambda}^{q}(x)\right)<\beta_{2} \delta_{0} K_{0} \tau^{t} \lambda^{(1 / t)-2} .
$$


When we apply (2.5.1) and (2.5.2) to this we get

$$
\beta \frac{1}{\delta} K_{0} \tau^{t} \lambda^{(1 / t)-2}<\frac{d}{d \lambda}\left(F_{\lambda}^{q}(x)\right)<\beta \delta K_{0} \tau^{t} \lambda^{(1 / t)-2} .
$$

Now let $K=\beta K_{0}$.

\section{Two Auxiliary Functions and an Associated Differential Equation}

In this section we consider a parameter value (say $\lambda=0$ ) at which some iterate of $F_{0}$, say $G_{0}=F_{0}^{q}-p$, is such that $\rho\left(G_{0}\right)=0$ but $\rho\left(G_{\lambda}\right)>0$ when $\lambda>0$. We assume that the function family $F_{\lambda}$ belongs to $\mathscr{F}^{\prime}$, so that the set $\left\{u_{i}\right\}$ of fixed points of $G_{0}$ constitutes a single cycle $(\bmod \mathbb{Z})$ of $F_{0}$. We assume $u_{i}<u_{i+1}$ for each $i$. We now define two functions on each interval $\left(u_{i}, u_{i+1}\right)$. As in property (iii') in the definition of the class $\mathscr{F}^{\prime}$ we let $t$ denote the lowest order non-vanishing derivative of $\phi_{0}(x)=G_{0}(x)-x$ at $u_{i}$.

Definition 3.1. For $z \in\left(u_{i}, u_{i+1}\right)$ we let

$$
R_{i}(z)=\lim _{k \rightarrow \infty}\left(G_{0}^{k}\right)^{\prime}\left(G_{0}^{-k}(z)\right) \cdot\left(G_{0}^{-k}(z)-u_{i}\right)^{t}
$$

and for $z \in\left(u_{i-1}, u_{i}\right)$, we let

$$
L_{i}(z)=\lim _{k \rightarrow \infty}\left(G_{0}^{k}\right)^{\prime}(z) /\left(G_{0}^{k}(z)-u_{i}\right)^{t} .
$$

The next two lemmas show that these limits exist, and prove some of the properties of $L_{i}$ and $R_{i}$.

\section{Lemma 3.2.}

(a) $R_{i}(z)$ is defined, continuous and non-negative, and the sequence defining $R_{i}(z)$ converges uniformly on any compact subset of $\left(u_{i}, u_{i+1}\right)$.

(b) $R_{i}(z)>0$ for $z$ sufficiently close to $u_{i}$.

(c) $R_{i}\left(G_{0}(z)\right)=G_{0}^{\prime}(z) \cdot R_{i}(z)$.

(d) $R_{i}(z)=0$ if and only if $z$ is in the forward orbit of a critical point of $G_{0}$.

(e) $R_{i}(z)$ is uniformly Lipschitz on any compact subset of $\left(u_{i}, u_{i+1}\right)$.

(f) $\int_{x}^{G_{0}(x)} \frac{1}{R_{i}(z)} d z=1$ for every $x \in\left(u_{i}, u_{i+1}\right)$.

Proof. We may assume that $u_{i}=0$. Let $\alpha=(t !) G_{0}^{(t)}(0)$. Let $\phi_{0}(x)=G_{0}(x)-x$ and let $z_{k}=G_{0}^{-k}(z)$. Using the Mean Value Theorem as in the proof of Lemma 2.1, we have for sufficiently large $n$, and for $k \geqq 0$,

$$
\frac{\phi_{0}\left(z_{n+1}\right)}{\phi_{0}\left(z_{n+k+1}\right)} \leqq \frac{\left(G_{0}^{n+k}\right)^{\prime}\left(z_{n+k}\right)}{\left(G_{0}^{n}\right)^{\prime}\left(z_{n}\right)} \leqq \frac{\phi_{0}\left(z_{n}\right)}{\phi_{0}\left(z_{n+k}\right)} \text {. }
$$

Thus

$$
\begin{aligned}
& \left|\left(z_{n+k}\right)^{t}\left(G_{0}^{n+k}\right)^{\prime}\left(z_{n+k}\right)-\left(z_{n}\right)^{t}\left(G_{0}^{n}\right)^{\prime}\left(z_{n}\right)\right| \\
& \quad=\left|\frac{z_{n+k}^{t}}{z_{n}^{t}} \cdot \frac{\left(G_{0}^{n+k}\right)^{\prime}\left(z_{n+k}\right)}{\left(G_{0}^{n}\right)^{\prime}\left(z_{n}\right)}-1\right| \cdot\left|\left(z_{n}\right)^{t}\left(G_{0}^{n}\right)^{\prime}\left(z_{n}\right)\right| \leqq A_{n, k}\left|\left(z_{n}\right)^{t}\left(G_{0}^{n}\right)^{\prime}\left(z_{n}\right)\right|,
\end{aligned}
$$


where

$$
A_{n, k}=\max \left\{\left|\left(\frac{z_{n+k}}{z_{n}}\right)^{t} \frac{\phi_{0}\left(z_{n+k}\right)}{\phi_{0}\left(z_{n+k+1}\right)}-1\right|, \quad\left|\left(\frac{z_{n+k}}{z_{n}}\right)^{t} \frac{\phi_{0}\left(z_{n}\right)}{\phi_{0}\left(z_{n+k}\right)}-1\right|\right\} .
$$

Since $\left(\phi_{0}\left(z_{1}\right) / \alpha z^{t}\right) \rightarrow 1$ as $z \rightarrow 0$, and $\left(z_{n+1} / z_{n}\right) \rightarrow 1$ as $n \rightarrow \infty$, (a) follows.

To prove (b) we note that by (2.4.9) we have for small $z$,

$$
\phi_{0}(z)=\{\alpha+Q(z)\} \cdot z^{t},
$$

where $Q(z) \rightarrow 0$ as $z \rightarrow 0$. Thus

$$
R_{i}(z)=\lim _{k \rightarrow \infty}\left(G_{0}^{k}\right)^{\prime}\left(z_{k}\right) \cdot \frac{\phi_{0}\left(z_{k}\right)}{\alpha+Q\left(z_{k}\right)} .
$$

Now letting $a_{m}=\phi_{0}\left(z_{m}\right)$, we have

$$
a_{1}<\frac{a_{1}}{a_{k+1}} \cdot a_{k}<\left(G_{0}^{k}\right)^{\prime}\left(z_{k}\right) \phi_{0}\left(z_{k}\right)<\frac{a_{0}}{a_{k}} a_{k}=a_{0} .
$$

Thus

$$
\frac{\phi_{0}\left(z_{1}\right)}{\alpha+Q\left(z_{k}\right)} \leqq z_{k}^{t}\left(G_{0}^{k}\right)^{\prime}\left(z_{k}\right) \leqq \frac{\phi_{0}(z)}{\alpha+Q\left(z_{k}\right)} .
$$

Letting $k \rightarrow \infty$ this gives

$$
\frac{\phi_{0}\left(G_{0}^{-1}(z)\right)}{\alpha} \leqq R_{i}(z) \leqq \frac{\phi_{0}(z)}{\alpha} .
$$

In other words,

$$
R_{i}(z)=\frac{\phi_{0}(\gamma(z))}{\alpha}
$$

for some $\gamma(z) \in\left[G_{0}^{-1}(z), z\right]$.

Parts (c) and (d) are easy consequences of (b) and the definition of $R_{i}(z)$.

To prove (e) we only need to prove it at $z \in\left(u_{i}, u_{i+1}\right)$ such that $G_{0}^{\prime}(y)>0$ for all $y \in(0, z)$, for then (c) allows us to extend the result to $\left(u_{i}, u_{i+1}\right)$. So suppose $G_{0}^{-1}\left(y_{2}\right)<y_{1}<y_{2}$ and let $U=\bigcup_{k \geqq 0} G_{0}^{-k}\left[y_{1}, y_{2}\right]$. Since for small $y, G_{0}^{\prime}(y)$ is monotonically increasing, we have meas $(U) \leqq A\left|y_{2}-y_{1}\right|$, where $A$ is a constant. Let $N f(y)$ denote the nonlinearity

$$
N f(y)=\frac{f^{\prime \prime}(y)}{f^{\prime}(y)} d y=d \log \left(f^{\prime}(y)\right)
$$

Therefore

$$
\int_{U} N G_{0}(y) d y \leqq B\left|y_{2}-y_{1}\right|
$$

for some constant $B$. Also

$$
N G_{0}^{k}(y)=\sum_{i=0}^{k-1}\left(G_{0}^{i}\right)^{*}\left(N G_{0}\right)(y),
$$


whence

$$
\int_{G_{0}^{-k}\left(y_{1}\right)}^{\sigma_{0}^{-k}\left(y_{2}\right)} N G_{0}^{k}(y) d y \leqq B\left|y_{2}-y_{1}\right| .
$$

Hence

$$
1 \leqq \frac{\left(G_{0}^{k}\right)^{\prime}\left(G_{0}^{-k}\left(y_{2}\right)\right)}{\left(G_{0}^{k}\right)^{\prime}\left(G_{0}^{-k}\left(y_{1}\right)\right)} \leqq e^{B\left|y_{2}-y_{1}\right|}
$$

That is, for some constant $C$ we have

$$
0 \leqq\left(G_{0}^{k}\right)^{\prime}\left(G_{0}^{-k}\left(y_{2}\right)\right)-\left(G_{0}^{k}\right)^{\prime}\left(G_{0}^{-k}\left(y_{1}\right)\right) \leqq C\left|y_{2}-y_{1}\right| \cdot\left(G_{0}^{k}\right)^{\prime}\left(G_{0}^{-k}\left(y_{1}\right)\right) .
$$

Hence

$$
\begin{aligned}
& \left|\left\{G_{0}^{-k}\left(y_{2}\right)\right\}^{t}\left(G_{0}^{k}\right)^{\prime}\left(G_{0}^{-k}\left(y_{2}\right)\right)-\left\{G_{0}^{-k}\left(y_{1}\right)\right\}^{t}\left(G_{0}^{k}\right)^{\prime}\left(G_{0}^{-k}\left(y_{1}\right)\right)\right| \\
& \quad \leqq\left(\left(G_{0}^{-k}\left(y_{2}\right)\right)^{t}-\left(G_{0}^{-k}\left(y_{1}\right)\right)^{t}\right)\left(G_{0}^{k}\right)^{\prime}\left(G_{0}^{-k}\left(y_{2}\right)\right)+C\left|y_{2}-y_{1}\right|\left(G_{0}^{-k}\left(y_{1}\right)\right)^{t}\left(G_{0}^{k}\right)^{\prime}\left(G_{0}^{-k}\left(y_{1}\right)\right) \\
& \quad=\left[1-\left(\frac{G_{0}^{-k}\left(y_{1}\right)}{G_{0}^{-k}\left(y_{2}\right)}\right)^{t}\right] \cdot\left(G_{0}^{-k}\left(y_{2}\right)\right)^{t}\left(G_{0}^{k}\right)^{\prime}\left(G_{0}^{-k}\left(y_{2}\right)\right)+C\left|y_{2}-y_{1}\right|\left(G_{0}^{-k}\left(y_{1}\right)\right)^{t}\left(G_{0}^{k}\right)^{\prime}\left(G_{0}^{-k}\left(y_{1}\right)\right) .
\end{aligned}
$$

Letting $k \rightarrow \infty$ this gives

$$
\left|R_{i}\left(y_{2}\right)-R_{i}\left(y_{1}\right)\right| \leqq C\left|y_{2}-y_{1}\right| R_{i}\left(y_{1}\right),
$$

from which (e) follows.
To prove part (f) we first show that $\int_{x}^{G_{0}(x)}\left(1 / R_{i}(z)\right) d z$ is independent of $x$. To do this we note that for small $x$,

$$
\frac{d}{d x} \int_{x}^{G_{0}(x)} \frac{1}{R_{i}(z)} d z=\frac{G_{0}^{\prime}(x)}{R_{i}\left(G_{0}(x)\right)}-\frac{1}{R_{i}(x)}=0
$$

by (c). To extend this result to all of $\left(u_{i}, u_{i+1}\right)$ we have by (c) that

$$
\int_{G_{0}(x)}^{G_{0}^{2}(x)} \frac{1}{R_{i}(w)} d w=\int_{x}^{G_{0}(x)} \frac{1}{R_{i}\left(G_{0}(z)\right)} G_{0}^{\prime}(z) d z=\int_{x}^{G_{0}(x)} \frac{1}{R_{i}(z)} d z,
$$

using the coordinate change $w=G_{0}(z)$.

To continue, we write $\phi_{0}(z)=\{\alpha+Q(z)\} \cdot z^{t}$ as before, and use (3.2.2) to see that for small $a$,

$$
\int_{a}^{G_{0}(a)} \frac{1}{R_{i}(z)} d z=\int_{a}^{G_{0}(a)} \frac{\alpha}{\phi_{0}(\gamma(z))} d z=\int_{a}^{a+\phi_{0}(a)} \frac{\alpha}{(\alpha+Q(\gamma(z))) \cdot \gamma(z)^{t}} d z .
$$

Now choose $a$ so small that $|Q(z)|<\varepsilon$ on $\left[a, G_{0}(a)\right]$ as well as $\alpha-\varepsilon<(\alpha+Q(\gamma(z)))$. $\left(\gamma(z)^{t} / z^{t}\right)<\alpha+\varepsilon$. Then

$$
\int_{a}^{a+(\alpha-\varepsilon) a^{t}} \frac{1}{(\alpha+\varepsilon) z^{t}} d z \leqq \int_{a}^{G_{0}(a)} \frac{1}{R_{i}(z)} d z \leqq \int_{a}^{a+(\alpha+\varepsilon) a^{t}} \frac{1}{(\alpha-\varepsilon) z^{t}} d z .
$$


That is,

$$
\begin{aligned}
\frac{\left(1+(\alpha-\varepsilon) a^{t-1}\right)^{t-1}-1}{(\alpha+\varepsilon)(t-1) a^{t-1}\left(1+(\alpha-\varepsilon) a^{t-1}\right)^{t-1}} & \leqq \int_{a}^{G_{0}(a)} \frac{1}{R_{i}(z)} d z \\
& \leqq \frac{\left(1+(\alpha+\varepsilon) a^{t-1}\right)^{t-1}-1}{(\alpha-\varepsilon)(t-1) a^{t-1}\left(1+(\alpha+\varepsilon) a^{t-1}\right)^{t-1}}
\end{aligned}
$$

Now let $a$ (and $\varepsilon) \rightarrow 0$ and find that

$$
\int_{a}^{G_{0}(a)} \frac{1}{R_{i}(z)} d z=1 .
$$

\section{Lemma 3.3.}

(a) $L_{i}(z)$ is well defined, continuous and non-negative, and the sequence defining $L_{i}(z)$ converges uniformly on any compact subset of $\left(u_{i-1}, u_{i}\right)$.

(b) $L_{i}(z)=G_{0}^{\prime}(z) L_{i}\left(G_{0}(z)\right)$.

(c) $\int_{x}^{G_{0}(x)} L_{i}(z) d z=1$ for every $x \in\left(u_{i-1}, u_{i}\right)$.

Proof. The proof is similar to the proof of Lemma 3.2, and is left to the reader.

We now introduce an ordinary differential equation on $\left(u_{i-1}, u_{i}\right)$ whose solutions play a very important role in the sequel.

Definition 3.4. For $a \in\left(u_{i-1}, u_{i}\right)$ and $z \in\left(u_{i}, u_{i+1}\right)$ we let $g_{z}:\left(u_{i-1}, u_{i}\right) \rightarrow\left(u_{i}, u_{i+1}\right)$ be the solution $g_{z}(x)=y(x)$ of the differential equation

$$
\frac{d y}{d x}=L_{i}(x) R_{i}(y(x))
$$

with initial condition $g_{z}(a)=z$. The fact that $a$ and $i$ have been suppressed in the notation will cause no difficulty in the sequel.

Lemma 3.5. For each choice of $i, a$, and $z, g_{z}$ is continuously differentiable increasing function with the property $g_{z}\left(G_{0}(x)\right)=G_{0}\left(g_{z}(x)\right)$.

Proof. The first two assertions are obvious. As to the last assertion, from Definition 3.4 it follows that

$$
\int_{g_{z}(x)}^{g_{\mathbf{z}}\left(G_{0}(x)\right)} \frac{d w}{R_{i}(w)}=\int_{x}^{G_{0}(x)} L_{i}(v) d v .
$$

But by Lemmas 3.2 and 3.3 we have

$$
\int_{g_{z}(x)}^{G_{0}\left(g_{z}(x)\right)} \frac{d w}{R_{i}(w)}=\int_{x}^{G_{0}(x)} L_{i}(v) d v=1 .
$$

Hence $G_{0}\left(g_{z}(x)\right)=g_{z}\left(G_{0}(x)\right)$.

We end this section by showing that the solution $y=g_{z}(x)$ of the differential Eq. (3.4) with initial condition $z=g_{z}(a)$ can be altered by arbitrarily small $C^{t}$ perturbations of $G_{0}$. What is important for us in the sequel is that if $r / s$ is Farey 
neighbour of $p / q(r q=p s \pm 1)$ and if $a=F_{0}^{s}(b) \mp r$, then the equality $g_{b} \circ\left(F_{0}^{s} \mp r\right)=\mathrm{Id}$ is generically false for a one parameter family $F_{\lambda}$.

Since $g_{z}$ and $G_{0}$ commute we may assume $b$ as close to a fixed point of $G_{0}$ as we please. For a fixed $m \in \mathbb{Z}_{+}$, suppose $\varepsilon(x)$ is an arbitrarily small $C^{t}$ perturbation of the identity function on $\left(G_{0}^{-m}(b), G_{0}^{-m+1}(b)\right)$ with the property $\varepsilon(x) \geqq x$ for all $x$ and $\varepsilon(x)>x$ for some $x$.

Let

$$
H_{0}(z)= \begin{cases}G_{0}(\varepsilon(z)) & \text { if } \quad z \in\left(G_{0}^{-m}(b), G_{0}^{-m+1}(b)\right) \\ G_{0}(z) & \text { elsewhere }\end{cases}
$$

It is easy to see that $H_{0}+p$ is the $q^{\text {th }}$ iterate of a similarly perturbed $F_{0}$. Let $R_{\varepsilon}(z)$ denote the function $R_{i}(z)$ constructed using $H_{0}$ in place of $G_{0}$, and let $R(z)$ denote $R_{i}(z)$ where for convenience we assume again that $u_{i}=0$. Then for $z \in\left[b, G_{0}(b)\right)$,

$$
R_{i}(z)=\lim _{k \rightarrow \infty}\left(H_{0}^{-k}(z)\right)^{t} \cdot\left(G_{0}^{k-m}\right)^{\prime}\left(H_{0}^{-k}(z)\right) \cdot\left(G_{0}^{m}\right)^{\prime}\left(G_{0}^{-m}((z)) \varepsilon^{\prime}\left(H_{0}^{-m}(z)\right) .\right.
$$

Now consider a point $z \in\left[b, G_{0}(b)\right)$ at which $\varepsilon^{\prime}\left(H_{0}^{-m}(z)\right) \leqq 1$ but $\varepsilon\left(H_{0}^{-m}(z)\right)>H_{0}^{-m}(z)$. Then $H_{0}^{-k}(z)<G_{0}^{-k}(z)$ and so (assuming $b$ sufficiently close to 0 ),

$$
\left(G_{0}^{k-m}\right)^{\prime}\left(H_{0}^{-k}(z)\right)<\left(G_{0}^{k-m}\right)^{\prime}\left(G_{0}^{-k}(z)\right),
$$

whence $R_{\varepsilon}(z)<R(z)$ at such points. But, if the differential equations

$$
\frac{d y}{d x}=L(x) R(y(x))
$$

and

$$
\frac{d y}{d x}=L(x) R_{\varepsilon}(y(x))
$$

have the same solution $y(x)$ with $y(a)=b$, then $R(y(x))=R_{\varepsilon}(y(x))$ for all $x \in\left[a, G_{0}(a)\right)$ and so $R=R_{\varepsilon}$ on $\left[b, G_{0}(b)\right)$ contradicting our findings. Thus the function $g_{b}$ has been altered by the perturbation of $F_{0}$. Finally, it should be noted that by choosing $m$ appropriately we can ensure that this perturbation of $F_{0}$ does not affect the restriction of $F_{0} \mp r$ to any particular given compact subinterval of $\left(u_{i}, u_{i+1}\right)$.

\section{The Return Maps}

In this section we will prove Proposition 4.1 and derive Proposition 1.3 from it. Proposition 4.1 establishes the asymptotically uniform behaviour for the iterates of $G_{\lambda}=F_{\lambda}^{q}-p$ at appropriately chosen parameter values. As always we assume that the family $F_{\lambda}$ belongs to the class $\mathscr{F}^{\prime}$, and that $\lambda_{0}$ is on the boundary of the set of parameter values $\lambda$ for which $\rho\left(F_{\lambda}\right)=p / q$. A Farey neighbour $r / s$ of $p / q$ is a rational number with $r q=p s \pm 1$. We will assume that $p / q<r / s$ and that $\rho\left(F_{\lambda}\right)>p / q$ for $\lambda>\lambda_{0}$. The case where the reverse inequality holds may be dealt with by replacing $F_{\lambda}(x)$ by $-F_{-\lambda}(-x)$. Suppose $u_{i-1}<u_{i}<u_{i+1}$ are adjacent points in the unique periodic orbit of $F_{\lambda_{0}}$. It is easy to see that $\left(F_{\lambda_{0}}^{s}-r\right)\left(u_{i}, u_{i+1}\right)=\left(u_{i-1}, u_{i}\right)$. We choose a fixed point $a \in\left(u_{i-1}, u_{i}\right)$. For each $z \in\left(u_{i}, u_{i+1}\right)$ and $n \in \mathbb{Z}_{+}$there is a unique 
parameter value $\lambda>\lambda_{0}$ such that $G_{\lambda}^{n}(a)=z$. Using that value of $\lambda$ we define $h_{n, z}=G_{\lambda}^{n}$.

Proposition 4.1 below proves the uniform convergence of the sequence of functions $h_{n, z}$ as $n \rightarrow \infty$. Since the return maps $k_{n, \lambda_{n}(w)}$ defined in Sect. 1 are related to the maps $h_{n, z}$ by the formula

$$
k_{n, \lambda_{n}(w)}=\left(F_{\lambda_{n}(w)}^{s}-r\right) \circ h_{n, z_{n}},
$$

where $z_{n}=\left(F_{\lambda_{n}(w)}^{s}-r\right)^{-1}(w)$, and since the functions $\left(F_{\lambda_{n}(w)}^{s}-r\right)$ clearly converge uniformly to $\left(F_{\lambda_{0}}^{s}-r\right)$, Proposition 1.3 is an immediate consequence of Proposition 4.1.

Proposition 4.1. Suppose $F_{\lambda}, u_{i}, a$ and $h_{n, z}$ are as defined above. Also suppose $z(\lambda) \in\left(u_{i}, u_{i+1}\right)$ varies continuously with $\lambda$. For a fixed $m \in \mathbb{Z}_{+}$let $I_{\lambda}=\left[a, G_{\lambda}^{m}(a)\right)$. Let $\lambda_{n}>\lambda_{0}, n=1,2,3, \ldots$ be a sequence of parameter values with $\lim \lambda_{n}=\lambda_{0}$ and for which $G_{\lambda_{n}}^{n}(a)=z\left(\lambda_{n}\right)$. Let $h_{n}=h_{n, z\left(\lambda_{n}\right)}$ and suppose I is a compact interval contained in $I_{\lambda}$ for all sufficiently small $\lambda$. Then the sequence $h_{n}$ converges to $g_{z(0)}$ uniformly on $I$.

Proof. For convenience we may assume that $\lambda_{0}=0$ and that $u_{i}=0$. We will write $L(x)$ and $R(y)$ in place of $L_{i}(x)$ and $R_{i}(y)$. Suppose $\delta>1$. By Lemma 2.1 there exist numbers $\tau>0$ and $\varepsilon>0$ such that $a<-\tau<0<\tau<z(\lambda)$ and $G_{\lambda}^{m+1}(-\tau)<0$ and $G_{\lambda}^{-m-1}(\tau)>0$ for all $\lambda \in(0, \varepsilon)$, and such that if $u \in\left[-\tau, G_{\lambda}^{m+1}(-\tau)\right)$ and $G_{\lambda}^{q}(u) \in\left[G_{\lambda}^{-m-1}(\tau), \tau\right)$, then

$$
\frac{1}{\delta}<\left(G_{\lambda}^{q}\right)^{\prime}(u)<\delta .
$$

On the other hand, it also follows from (3.2.2) and the corresponding formula for $L(z)$ for $z$ near 0 :

$$
L(z)=\frac{\alpha}{\phi_{0}(\gamma(z))},
$$

that with $u$ and $v$ as described above, and $\varepsilon$ small enough,

$$
\frac{1}{\delta}<L(u) R(v)<\delta .
$$

We may assume $\varepsilon$ so small that for some fixed integers $k$ and $l$,

$$
G_{\lambda}^{k}(a) \in\left[-\tau, G_{\lambda}(-\tau)\right)
$$

and

$$
G_{\lambda}^{-l}(z(\lambda)) \in\left[G_{\lambda}^{-m-1}(\tau), G_{\lambda}^{-m}(\tau)\right)
$$

for all $\lambda \in(0, \varepsilon)$. Then for any $x \in I_{\lambda}$ and $\left.y \in J_{\lambda}=\left[z(\lambda), G_{\lambda}^{m}(z)\right)\right)$ we have

$$
G_{\lambda}^{k}(x) \in\left[-\tau, G_{\lambda}^{m+1}(-\tau)\right)
$$

and

$$
G_{\lambda}^{-l}(y) \in\left[G_{\lambda}^{-m-1}(\tau), \tau\right) .
$$


Now consider $h_{n \mid I}$. Suppose $h_{n}(x)=y$. Then using 2.1,

$$
\frac{1}{\delta}\left(G_{\lambda_{n}}^{k}\right)^{\prime}(x)\left(G_{\lambda_{n}}^{l}\right)^{\prime}\left(G_{\lambda_{n}}^{-l}(y)\right) \leqq h_{n}^{\prime}(x) \leqq \delta\left(G_{\lambda_{n}}^{k}\right)^{\prime}(x)\left(G_{\lambda_{n}}^{l}\right)^{\prime}\left(G_{\lambda_{n}}^{-l}(y)\right) .
$$

Since $G_{\lambda_{n}}$ converges to $G_{0}$ in the $C^{1}$ topology, it follows from this that for sufficiently large $n$,

$$
\frac{1}{\delta^{2}}\left(G_{0}^{k}\right)^{\prime}(x)\left(G_{0}^{l}\right)^{\prime}\left(G_{0}^{-l}(y)\right)<h_{n}^{\prime}(x)<\delta^{2}\left(G_{0}^{k}\right)^{\prime}(x)\left(G_{0}^{l}\right)^{\prime}\left(G_{0}^{-l}(y)\right)
$$

Since

$$
L(x) R(y)=\left(G_{0}^{k}\right)^{\prime}(x) L\left(G_{0}^{k}(x)\right) R\left(G_{0}^{-l}(y)\right)\left(G_{0}^{l}\right)^{\prime}\left(G_{0}^{-l}(y)\right),
$$

it follows from (4.1.2) that

$$
\frac{1}{\delta^{3}} L(x) R(y)<h_{n}^{\prime}(x)<\delta^{3} L(x) R(x) .
$$

Substracting from this the equation

$$
g_{z\left(\lambda_{n}\right)}^{\prime}(x)=L(x) R\left(g_{z\left(\lambda_{n}\right)}(x)\right)
$$

and writing $g_{n}$ for $g_{z\left(\lambda_{n}\right)}$, we get

$$
L(x)\left(\frac{1}{\delta^{3}} R\left(h_{n}(x)\right)-R\left(g_{n}(x)\right)\right)<h_{n}^{\prime}(x)-g_{n}^{\prime}(x)<L(x)\left(\delta^{3} R\left(h_{n}(x)\right)-R\left(g_{n}(x)\right)\right) .
$$

Thus,

$$
\begin{aligned}
L(x) & \left\{\left(\frac{1}{\delta^{3}} R\left(h_{n}(x)\right)-R\left(g_{n}(x)\right)\right)+\left(\frac{1}{\delta^{3}}-1\right) R\left(g_{n}(x)\right)\right\} \\
& <h_{n}^{\prime}(x)-g_{n}^{\prime}(x)<L(x)\left\{\left(\delta^{3} R\left(h_{n}(x)\right)-R\left(g_{n}(x)\right)\right)+\left(\delta^{3}-1\right) R\left(g_{n}(x)\right)\right\} .
\end{aligned}
$$

Therefore, using Lemma 3.2(e),

$$
\left|h_{n}(x)-g_{n}(x)\right|^{\prime}<\delta^{3} L(x) C\left|h_{n}(x)-g_{n}(x)\right|+\left(\delta^{3}-1\right) L(x) R\left(g_{n}(x)\right) .
$$

By Gronwall's inequality this implies that for sufficiently large $n$,

$$
\left|h_{n}(x)-g_{n}(x)\right| \leqq \frac{\left(\delta^{3}-1\right) N}{M}\left(e^{M|x-a|}-1\right),
$$

where $M$ is an upper bound for $\delta^{3} L(x)$ on $I$ and where $N$ is a uniform upper bound (independent of $n$ ) for $L(x) R\left(g_{n}(x)\right)$ on $I$. Since $\delta$ may be chosen as close to 1 as we wish, this proves that on $I$ we have $\left\|h_{n}-g_{n}\right\| \rightarrow 0$ as $n \rightarrow \infty$. A similar argument shows that $g_{n}$ converges uniformly to $g_{z(0)}$. These combine to prove the proposition.

\section{Variation of the Return Maps}

In this section we will derive Proposition 1.4 which estimates the derivative of the return map with respect to the parameter $\lambda$. In fact we will prove a more general result below, which applies to a function family belonging to the larger class $\mathscr{F}^{\prime}$. 
Let $\lambda_{0}, p / q, G_{\lambda}$, and $\left\{u_{i}\right\}$ be defined as in the preceding section, and let $z(\lambda)=G_{\lambda}^{n}(a)$ for a fixed point $a \in\left(u_{i-1}, u_{i}\right)$. We will study the function $z(\lambda)$ for values of $\lambda$ near $\lambda_{0}$.

As in Lemma 2.4 we assume that $t$ is the smallest integer larger than 1 for which $G_{\lambda_{0}}^{(t)}\left(u_{i}\right) \neq 0$, and we let

$$
\begin{aligned}
(\mathrm{t} !) \alpha_{i} & =G_{\lambda_{0}}^{(t)}\left(u_{i}\right), \quad \beta_{i}=\frac{d}{d \lambda} G_{\lambda}\left(u_{i}\right)_{\mid \lambda=\lambda_{0}}, \\
L_{t} & =\lim _{\lambda \rightarrow 0^{+}} \lambda^{2-(1 / t)} \int_{-\tau}^{t} \frac{d x}{\left(\lambda+x^{t}\right)^{2}},
\end{aligned}
$$

and

$$
K_{i}=L_{t} \cdot\left(\frac{\alpha_{i}}{\beta_{i}}\right)^{1-(1 / t)} .
$$

The function $z(\lambda)$ is described in the following result:

Proposition 5.1. Let $J$ be a compact subinterval of $\left(u_{i-1}, u_{i}\right)$. Then for sufficiently large $n$ there is a function $K_{i}(n, z)>0$ defined on a neighbourhood of $J$ such that if $G_{\lambda}^{n}(a)=z \in J$, then $(d / d \lambda) G_{\lambda}^{n}(a)=K_{i}(n, z) \lambda^{(1 / t)-2}$. Furthermore, $\lim _{n \rightarrow \infty} K_{i}(n, z)=K_{i} \cdot R_{i}(z)$, uniformly on $J$.

In the case $t=2$ this gives $(d / d \lambda) G_{\lambda}^{n}(a)=K_{i}(n, z) \lambda^{-3 / 2}$. Since the return map $k_{n, \lambda}$ is equal to

$$
k_{n, \lambda_{n}(w)}=\left(F_{\lambda_{n}(w)}^{s}-r\right) \circ G_{\lambda_{n}(w)}^{n},
$$

and since $\left(F_{\lambda_{n}(w)}^{s}-r\right)$ converges uniformly to $\left(F_{\lambda_{0}}^{s}-r\right)$ in the $C^{1}$-topology, Proposition 1.4 is clearly an immediate consequence.

Proof. We will assume that $\lambda_{0}=0, i=0$, and $u_{i}=0$, and we will suppress the subscript on $K_{i}, K_{i}(n, z)$, and $R_{i}(z)$. Suppose $\delta>1$ and $v>0$ are given and choose $c \in\left(u_{0}, u_{1}\right)$ and $m>2$ such that $J \subseteq\left[c, G_{0}^{m-2}(c)\right]$. Obtain numbers $\tau$ and $\varepsilon$ for which the conclusions of Lemmas 2.2 and 2.5 are satisfied and for which we have as well that

$$
\frac{1}{\delta}<\frac{\tau}{z}<\delta
$$

for all $z \in\left[G_{0}^{-m}(\tau), \tau\right)$. Let $k, l \in \mathbb{Z}_{+}$be such that

$$
G_{0}^{k}(a) \in\left[-\tau, G_{0}^{m-1}(-\tau)\right)
$$

and

$$
G_{0}^{-l}(c) \in\left[G_{0}^{-m+1}(\tau), G_{0}^{-m+2}(\tau)\right) .
$$

By continuity we may assume that

$$
G_{\lambda}^{k}(a) \subseteq\left[-\tau, G_{0}^{m}(-\tau)\right)
$$

and

$$
G_{\lambda}^{-l}(J) \subseteq\left[G_{0}^{-m}(\tau), \tau\right)
$$


for all $\lambda \in(0, \varepsilon)$. Next we may first assume $\tau$ so small, and therefore $l$ so large that on $J$ the $C^{0}$-norm of

$$
R(z)-\left(G_{0}^{-l}(z)\right)^{t}\left(G_{0}^{l}\right)^{\prime}\left(G_{0}^{-l}(z)\right)
$$

is smaller than $v$, and then assume that $\varepsilon$ is so small that for all $\lambda \in(0, \varepsilon)$, the $C^{0}$-norm on $J$ of

$$
\left(G_{\lambda}^{-l}(z)\right)^{t}\left(G_{\lambda}^{l}\right)^{\prime}\left(G_{\lambda}^{-l}(z)\right)-\left(G_{0}^{-l}(z)\right)^{t}\left(G_{0}^{l}\right)^{\prime}\left(G_{0}^{-l}(z)\right)
$$

is also less than $v$. Then if $G_{\lambda}^{n}(a)=z \in J$ we have

$$
\begin{aligned}
\frac{d}{d \lambda} G_{\lambda}^{n}(a)= & \frac{\partial G_{\lambda}^{l}}{\partial \lambda}\left(G_{\lambda}^{n-l}(a)\right)+\left(G_{\lambda}^{l}\right)^{\prime}\left(G_{\lambda}^{n-l}(a)\right) \cdot \frac{\partial G_{\lambda}^{n-l-k}}{\partial \lambda}\left(G_{\lambda}^{k}(a)\right) \\
& +\left(G_{\lambda}^{l}\right)^{\prime}\left(G_{\lambda}^{n-l}(a)\right) \cdot\left(G_{\lambda}^{n-l-k}\right)^{\prime}\left(G_{\lambda}^{k}(a)\right) \cdot \frac{\partial G_{\lambda}^{k}}{\partial \lambda}(a) .
\end{aligned}
$$

Now note that $\partial G_{\lambda}^{l} / \partial \lambda$ is bounded uniformly on $G_{\lambda}^{-l}\left(J_{0}\right)$, that $\left(G_{\lambda}^{l}\right)^{\prime}\left(G_{\lambda}^{n-l}(a)\right)$ converges to $\left(G_{0}^{l}\right)^{\prime}\left(G_{0}^{-l}(z)\right)$ as $n \rightarrow \infty$, and that $\left(\partial G_{\lambda}^{k} / \partial \lambda\right)(a)$ is uniformly bounded for small $\lambda$. Furthermore, $\left(G_{\lambda}^{n-l-k}\right)^{\prime}\left(G_{\lambda}^{k}(a)\right)$ is uniformly bounded by Lemma 2.4 , while by Lemma 2.5 we have

$$
\frac{1}{\delta} K \tau^{t} \lambda^{(1 / t)-2}<\frac{\partial G_{\lambda}^{n-l-k}}{\partial \lambda}\left(G_{\lambda}^{k}(a)\right)<\delta K \tau^{t} \lambda^{(1 / t)-2} .
$$

Using these facts along with (5.1.1) we get

$$
B_{1}+\frac{1}{\delta^{3}}(R(z)-2 v) K \lambda^{(1 / t)-2}<\frac{d}{d \lambda} G_{\lambda}^{n}(a)<B_{1}+\delta^{3}(R(z)+2 v) K \lambda^{(1 / t)-2},
$$

where $B_{1}$ is a fixed constant independent of $n$ (but not of $\tau$ ). Thus, letting $K(n, z)=\lambda^{2-(1 / t)}(d / d \lambda) G_{\lambda}^{n}(a)$,

$$
B_{1} \lambda^{2-(1 / t)}+\frac{1}{\delta^{3}}(K R(z)-2 v K)<K(n, z)<B_{1} \lambda^{2-(1 / t)}+\delta^{3}(K R(z)+2 v K) .
$$

Hence,

$$
|K(n, z)-K R(z)|<\left(\delta^{3}-1\right) K R(z)+2 \delta^{3} v K+B_{1} \lambda^{2-(1 / t)} .
$$

With $N_{n}=\sup _{z \in J}|K(n, z)-K R(z)|$, and letting $n \rightarrow \infty$, this implies that

$$
\limsup _{n \rightarrow \infty} N_{n} \leqq\left(\delta^{3}-1\right) K\left\{\sup _{z \in J} R(z)\right\}+2 \delta^{3} v K .
$$

Since $\left(\delta^{3}-1\right)$ and $v$ can be chosen arbitrarily small, this proves the result.

\section{Proofs of the Main Theorems}

In this section we complete the paper by proving Theorems 1.1 and 1.2. We restrict our function families to the generic class $\mathscr{F}$ as indicated in the hypotheses of these theorems. We leave it to the reader to formulate the corresponding results for 
families belonging to $\mathscr{F}^{\prime}$. Their proofs are obvious generalizations of the proofs presented below.

The idea is to use the fact that the return maps $h_{n, z}=G_{\lambda}^{n}$ converges uniformly (Proposition 4.1) and that the dependence of $z$ on $\lambda$ can be estimated (Proposition 5.1) to obtain an approximate value for the widths of the parameter intervals between the times at which the graphs of of $\left\{F_{\lambda}^{s}-r\right\}^{-1}$ and $h_{n, z}=G_{\lambda}^{n}$ meet at a certain point (giving Theorem 1.1) as well as the widths of the parameter intervals over which the graph of $h_{n, z}=G_{\lambda}^{n}$ crosses the graph of $\left\{F_{\lambda}^{s}-r\right\}^{-1}$ (giving Theorem 1.2).

For the proofs of Theorems 1.1 and 1.2 we may once again introduce the simplifications we have used repeatedly: $\lambda_{0}=0$ will be the largest parameter value at which $\rho\left(F_{\lambda}\right)=p / q, G_{\lambda}$ will denote $F_{\lambda}^{q}-p$, and $u_{-1}<0<u_{1}$ are three consecutive fixed points of $G_{0}$. We will also let $H_{\lambda}$ denote $F_{\lambda}^{s}-r$.

Proof of Theorem 1.1. Since $F_{0}$ maps $\left(u_{i-1}, u_{i}\right)$ to $\left(u_{i+p-1}, u_{i+p}\right)$, and since $F_{i}$ is uniformly close to $F_{0}$ for small $\lambda$, we may assume that $a \in\left(u_{-1}, 0\right)$. Let $b=H_{0}^{-1}(a)$ and let $H_{\lambda_{n}}^{-1}(a)=b_{n}$. Then $\lim b_{n}=b, \lim G_{\lambda_{n}}\left(b_{n}\right)=G_{0}(b)$, and $G_{\lambda_{n}}^{n}(a)=b_{n}$. For the moment let us assume that $G_{0}$ has no critical points in $\left[0, G_{0}^{2}(b)\right]$. Let $\delta>1$. Then for sufficiently large $n$,

$$
\begin{aligned}
& \delta\left(G_{0}(b)-b\right)>G_{\lambda_{n}}^{n+1}(a)-G_{\lambda_{n+1}}^{n+1}(a) \\
& \quad=\int_{\lambda_{n+1}}^{\lambda_{n}} \frac{d}{d \lambda} G_{\lambda}^{n+1}(a) d \lambda=\int_{\lambda_{n+1}}^{\lambda_{n}} K_{i}\left(n+1, G_{\lambda}^{n+1}(a)\right) \lambda^{-3 / 2} d \lambda>2 M_{1}\left(\frac{1}{\sqrt{\lambda_{n+1}}}-\frac{1}{\sqrt{\lambda_{n}}}\right),
\end{aligned}
$$

where $M_{1}>0$ is a strict lower bound for the value of $K_{i} R_{i}(z)$ on $\left[b, G_{0}^{2}(b)\right]$; the last step uses Proposition 5.1. Similarly,

$$
G_{0}(b)-b<2 M_{2}\left(\frac{1}{\sqrt{\lambda_{n+1}}}-\frac{1}{\sqrt{\lambda_{n}}}\right),
$$

where $M_{2}>0$ is a strict upper bound of the value of $K_{i} R_{i}(z)$ on $\left[b, G_{0}^{2}(b)\right]$. Thus, letting

$$
A_{1}=\frac{1}{2 M_{2}}\left(G_{0}(b)-b\right), \quad A_{2}=\frac{\delta}{2 M_{1}}\left(G_{0}(b)-b\right)
$$

and

$$
c=\frac{1}{\sqrt{\lambda_{n}}}
$$

we have for $m>n$,

$$
(m-n) A_{1}+c<\frac{1}{\sqrt{\lambda_{n}}}<(m-n) A_{2}+c .
$$

Therefore,

$$
\frac{1}{\left((m-n) A_{2}+c\right)^{2}}<\lambda_{m}<\frac{1}{\left((m-n) A_{1}+c\right)^{2}} .
$$


This proves the theorem for the case where $G_{0}$ has no critical point on $\left[0, G_{0}^{2}(b)\right]$.

If $G_{0}$ does have a critical point on $\left[0, G_{0}^{2}(b)\right]$, replace $a$ by $a_{1}=G_{0}^{-k}(a)$ and let $b_{1}=G_{0}^{-k}(b)=H_{0}^{-1}\left(a_{1}\right)$, where $k$ is large enough to ensure that $G_{0}$ has no critical points on $\left[0, G_{0}^{2}\left(b_{1}\right)\right]$. Let $v_{n}$ be the parameter value for which $H_{v_{n}} G_{v_{n}}\left(a_{1}\right)=a_{1}$, so that

$$
\frac{1}{\left((m-n) A_{2}+c\right)^{2}}<v_{m}<\frac{1}{\left((m-n) A_{1}+c\right)^{2}}
$$

by the proof so far. But for sufficiently large $n$ we have $G_{0}^{k}\left(a_{1}\right)>G_{v_{n}}^{k-1}\left(a_{1}\right)$. Therefore,

$$
H_{v_{n}} G_{v_{n}}^{n+1}(a)=H_{v_{n}} G_{v_{n}}^{n+1}\left(G_{0}^{k}\left(a_{1}\right)\right)>H_{v_{n}} G_{v_{n}}^{n+k}\left(a_{1}\right)=G_{v_{n}}^{k}\left(a_{1}\right)>G_{0}^{k}\left(a_{1}\right)=a,
$$

whence $v_{n}>\lambda_{n+1}$. Similarly,

$$
H_{v_{n}} G_{v_{n}}^{n-1}(a)=H_{v_{n}} G_{v_{n}}^{n-1}\left(G_{0}^{k}\left(a_{1}\right)\right)<H_{v_{n}} G_{v_{n}}^{n+k-1}\left(a_{1}\right)=G_{v_{n}}^{k-1}\left(a_{1}\right)<G_{0}^{k}\left(a_{1}\right)=a .
$$

Thus $v_{n}<\lambda_{n-1}$. Combining these two results we get $v_{m+1}<\lambda_{m}<v_{m-1}$, from which we obtain

$$
\frac{1}{\left((m-n+1) A_{2}+c\right)^{2}}<\lambda_{m}<\frac{1}{\left((m-n-1) A_{1}+c\right)^{2}} .
$$

From this the result follows immediately.

Before we prove Theorem 1.2 we need a lemma to show that $H_{\lambda}^{-1}=\left\{F_{\lambda}^{s}-r\right\}^{-1}$ converges uniformly on any given compact subinterval of $\left(u_{i-1}, u_{i}\right)$. The result is simple, and undoubtedly well known, but I do not know a reference for it.

Lemma 6.1. Suppose $f$ and $g_{\lambda}$ are homeomorphisms on a compact subinterval of $\mathbb{R}$ and that $g_{\lambda} \rightarrow f$ uniformly as $\lambda \rightarrow 0^{+}$. Then on any compact interval I contained in the image of $g_{\lambda}$ for all sufficiently small $\lambda, g_{\lambda}^{-1}$ converges uniformly to $f^{-1}$.

Proof. First we note that if for $\varepsilon_{1}>0$ we let

$$
\omega\left(\varepsilon_{1}\right)=\sup \left\{|x-y|:|f(x)-f(y)|=\varepsilon_{1}\right\},
$$

then $\omega\left(\varepsilon_{1}\right) \rightarrow 0^{+}$as $\varepsilon_{1} \rightarrow 0^{+}$. We may assume that $f$ is increasing. Now suppose $\varepsilon_{2}>0$ is given. Choose $\varepsilon_{1}>0$ so that $\omega\left(\varepsilon_{1}\right)<\varepsilon_{2}$. Then for any $y \in I$ and $x=f^{-1}(y)$, $f\left(x-\omega\left(e_{1}\right)\right) \leqq f(x)-\varepsilon_{1}$ and $f\left(x+\omega\left(e_{1}\right)\right) \geqq f(x)+\varepsilon_{1}$. Now choose $\varepsilon>0$ so small that for $\lambda \in(0, \varepsilon),\left\|g_{\lambda}-f\right\|<\varepsilon_{1}$. Then $g_{\lambda}\left(x-\omega\left(\varepsilon_{1}\right)\right)<f(x)$ and $g_{\lambda}\left(x+\omega\left(\varepsilon_{1}\right)\right)<f(x)$. Hence, if $g_{\lambda}(u)=f(x)$, then $u \in\left(x-\omega\left(\varepsilon_{1}\right), x+\omega\left(\varepsilon_{1}\right)\right) \subseteq\left(x-\varepsilon_{2}, x+\varepsilon_{2}\right)$. That is,

$$
\left|g_{\lambda}^{-1}(y)-f^{-1}(y)\right|<\varepsilon_{2} \text {. }
$$

Proof of Theorem 1.2. Choose $b \in\left(0, u_{1}\right)$ so that $G_{0}$ has no critical point in $\left[0, G_{0}^{2}(b)\right]$. Let $a=H_{0}^{-1}(b)$ and let $I=\left[a, G_{0}^{2}(a)\right]$. By Lemma $6.1, H_{\lambda}$ converges uniformly on $I$ to $H_{0}$ as $\lambda \rightarrow 0^{+}$. For $z \in\left[G_{0}^{-1}(b), G_{0}(b)\right]$, let $\lambda_{n}(z)$ be such that $h_{n, z}(a)=G_{\lambda_{n}(z)}^{n}(a)=z$. By Proposition 5.1, $h_{n, z}$ converges uniformly on $I$ to the function $g_{z}$ defined in 3.4. Furthermore, it follows from the definition of $g_{z}$ that if $z_{1}<z_{2}$, then $g_{z_{1}}(x)<g_{z_{2}}(x)$ for all $x \in I$, and Lemma 3.5 we have $g_{z}\left(G_{0}(a)\right)=G_{0}(b)$. Now $\rho\left(F_{\lambda}\right)=\theta_{n}=(n p+r) /(n q+s)$ if and only if there is a point $z_{0}$ such that $G_{\lambda}^{n} \circ\left(F_{\lambda}^{s}-r\right)\left(z_{0}\right)=z_{0}$; that is, if and only if there is a point $x_{0}=\left(F_{\lambda}^{s}-r\right)\left(z_{0}\right)$ such 
that $G_{\lambda}^{n}\left(x_{0}\right)=H_{\lambda}\left(x_{0}\right)$. But this is the case if and only if there is such a point $x_{0}$ in the interval $\left[a, G_{\lambda}(a)\right]$. Since $\left[a, G_{\lambda}(a)\right] \subseteq I$ for small $\lambda$, we conclude that $\rho\left(F_{\lambda}\right)=\theta_{n}$ if and only if the graphes of $H_{\lambda}$ and $G_{\lambda}^{n}$ meet on $I$.

Let $z_{1} \in\left[G_{0}^{-1}(b), b\right]$ be the smallest number $z$ such that the graph of $g_{z}$ meets the graph of $H_{0}$ (note that $g_{b}(a)=b=H_{0}(a)$ ), and let $z_{2} \in\left[b, G_{\lambda}(b)\right.$ ) be the largest. By the discussion at the end of Sect. 3 we know that for the generic family $F_{\lambda}$ we have $z_{1}<z_{2}$. Choose $w_{1}$ and $w_{2}$ so that $z_{1}<w_{1}<b<w_{2}<z_{2}$. Let $\eta>0$ be such that

$$
3 \eta<\inf \left\{\inf _{z \in I}\left|H_{0}-g_{w_{1}}\right|, \inf _{z \in I}\left|H_{0}-g_{w_{2}}\right|\right\} .
$$

Choose $N \in \mathbb{Z}_{+}$so that for $n \geqq N$, on the interval $I$,

$$
\left\|H_{\lambda}-H_{0}\right\|<\eta, \quad\left\|h_{n, w_{1}}-g_{w_{1}}\right\|<\eta, \quad\left\|h_{n, w_{2}}-g_{w_{2}}\right\|<\eta \text {. }
$$

Thus, for $n \geqq N, h_{n, w_{1}}<H_{\lambda}$ and $h_{n, w_{2}}>H_{\lambda}$. In particular, the parameter interval on which $\rho\left(F_{\lambda}\right)=\theta_{n}$ is contained in the interval $\left(\lambda_{n}\left(w_{1}\right), \lambda_{n}\left(w_{2}\right)\right)$. We estimate the size of this interval now:

$$
\begin{aligned}
& w_{2}-w_{1}=G_{\lambda_{n}\left(w_{2}\right)}^{n}(a)-G_{\lambda_{n}\left(w_{1}\right)}^{n}(a), \\
& \int_{\lambda_{n}\left(w_{1}\right)}^{\lambda_{n}\left(w_{2}\right)} \frac{d}{d \lambda} G_{\lambda}^{n}(a) d \lambda=\int_{\lambda_{n}\left(w_{1}\right)}^{\lambda_{n}\left(w_{2}\right)} K_{i}\left(n . G_{\lambda}^{n}(a)\right) \lambda^{-3 / 2} d \lambda .
\end{aligned}
$$

Choosing $M_{1}>0$ and $M_{2}>0$ so that $M_{1}<K_{i} R_{i}(z)<M_{2}$ for all $z \in\left[w_{1}, w_{2}\right]$, we obtain from this,

$$
2 M_{2}\left(\frac{1}{\sqrt{\lambda_{n}\left(w_{1}\right)}}-\frac{1}{\sqrt{\lambda_{n}\left(w_{2}\right)}}\right) \geqq w_{2}-w_{1},
$$

whence

$$
\frac{1}{\sqrt{\lambda_{n}\left(w_{1}\right)}}-\frac{1}{\sqrt{\lambda_{n}\left(w_{2}\right)}} \geqq \frac{w_{2}-w_{1}}{2 M_{2}} .
$$

Therefore,

$$
\Delta_{n} \geqq \lambda_{n}\left(w_{2}\right)-\lambda_{n}\left(w_{1}\right) \geqq \frac{w_{2}-w_{1}}{2 M_{2}}\left(\sqrt{\lambda_{n}\left(w_{1}\right) \lambda_{n}\left(w_{2}\right)}\right)\left(\sqrt{\lambda_{n}\left(w_{1}\right)}+\sqrt{\lambda_{n}\left(w_{2}\right)}\right) .
$$

But by Theorem 1.1 we have

$$
C_{1}(n q+s)^{-2}<\lambda_{n}\left(w_{2}\right), \quad \lambda_{n}\left(w_{1}\right)<C_{2}(n q+s)^{-2}
$$

for some positive constants $C_{1}$ and $C_{2}$. Thus

$$
\Delta_{n}>C_{3}(n q+s)^{-3}
$$

for a positive constant $C_{3}$. If we repeat this argument using two numbers $w_{1}$ and $w_{2}$ such that $w_{1}<z_{1}<z_{2}<w_{2}$ we get the inequality

$$
\Delta_{n}<C_{4}(n q+s)^{-3} \text {. }
$$


Acknowledgements. The author would like to acknowledge helpful conversations with J. Davis, R. D. Norman, and G. Swiątek. This research was supported by the Natural Sciences and Engineering Research Council of Canada

\section{References}

1. Alstr $\phi \mathrm{m}$, P.: Map dependence of the fractal dimension deduced from iteration of circle maps. Commun. Math. Phys. 104, 581-589 (1986)

2. Arnol'd, V. I.: Small denominators I. Mappings of the circumference onto itself. Am. Math. Soc. Transl. 46, 213-284 (1965)

3. Cvitanović, P., Shraiman, B., Söderberg, B.: Physica Scripta 32, 263-270 (1985)

4. Ecke, R. E., Farmer, J. D., Umberger, D. K.: Scaling of the Arnol'd tongues. Nonlinearity 2, 175-196 (1989)

5. Graczyk, J.: Harmonic scaling for smooth families of diffeomorphisms of the circle, preprint, Institute of Mathematics, University of Warsaw, PKIN, 9th floor, 00-901 Warsaw

6. Herman, M. R.: Mésure de Lebesque et nombre de rotation. In: Geometry and Topology, Palis, J., do Carmo, M. (eds.). Springer Lecture Notes in Mathematics, vol. 597, pp. 271-293. Berlin, Heidelberg, New York: Springer 1977

7. Jensen, M. H., Bak, P., Bohr, T.: Complete devil's staircase, fractal dimension and universality of mode-locking structure in the circle map. Phys. Rev. Lett. 50, 1637-1639 (1983)

8. Jonker, L., Rand, D. A.: Universal properties of maps of the circle with $\varepsilon$-singularities. Commun. Math. Phys. 90, 273-292 (1983)

9. Poincaré, H.: Mémoire sur les courbes définies par les équations differentielles I-IV, Oeuvres Complétes, t.1. Paris: Gauthier-Villars 1952

10. Rand, D., Ostlund, S., Sethna, J., Siggia, E. D.: A universal transition from quasi-periodicity to chaos in dissipative systems. Phys. Rev. Lett. 49, 132-135 (1982)

11. Swiątek, G.: Rational rotation numbers for maps of the circle. Commun. Math. Phys. 119, 109-128 (1988)

Communicated by J.-P. Eckmann

Received February 6, 1989 
